\title{
Effect of a Design Discharge Planning Program for Stroke Patients on Their Quality of Life and Activity of Daily Living
}

\author{
Amal Said Taha ${ }^{1} \&$ Rawia Ali Ibrahim ${ }^{1}$ \\ ${ }^{1}$ Medical Surgical Nursing, Faculty of Nursing, Benha University, Egypt \\ Correspondence: Amal Said Taha, Assistant Professor of Medical Surgical Nursing, Faculty of Nursing, Benha \\ University, Egypt.
}

Received: January 29, 2020

Accepted: February 26, 2020

Online Published: March 17, 2020

doi:10.20849/ijsn.v5i1.724

URL: https://doi.org/10.20849/ijsn.v5i1.724

\begin{abstract}
Background: Stroke is a debilitating neurological disorder, which causes multiple physical and psychosocial challenges to people surviving a stroke. Effective discharge planning program is an active process beginning in hospitalization care and has a goal of returning to the community and aims at assisting the patients to recapture their functional independence and to return to an active and productive lifestyle. Aim: was to evaluate effect of a design discharge planning program for stroke patients on their quality of life and activity of daily living. Research design: Quasi experimental design was utilized to fulfill the aim of this study. Setting: The study was conducted at the stroke department and stroke outpatient clinics affiliated to Benha University Hospital. Sample: A convenience sample of 50 patients admitted at the above mentioned setting and diagnosed with stroke. Tools: Three tools were used: I-Patients Interviewing questionnaire sheet; II-Stroke Specific Quality of Life Scale and III- Barthel Index for Activities of Daily Living. Results: the present study revealed that (a) the mean total and subtotal knowledge scores of stroke patients were increased immediately after implementing a design discharge planning program with statistical significant difference compared to pre implementation. (b) The mean total and subtotal quality of life scores were higher after implementing discharge planning program with a high statistical significant difference compared to pre implementation. (c) The mean total and subtotal activity of daily living scores were higher after implementing discharge planning program with a high statistical significant difference compared to pre implementation. (d) There were a positive correlation between patients' knowledge, quality of life and activity of daily living with a statistical significant difference. So, all research hypotheses of the study were supported. Conclusion: Applying a design discharge planning program had statistically significant improvement on knowledge, quality of life and activity of daily living for studied patients. Recommendations: replication of this study on a larger probability sample and evaluating its impact on health outcomes among stroke patients.
\end{abstract}

Keywords: design discharge planning program, quality of life, activity of daily living, stroke

\section{Introduction}

Stroke is a condition that can generally be understood as a form of "brain attack" in which continuous supply of blood to an area of the brain is interrupted (National stroke association, 2016). Stroke is a debilitating neurological disorder, which causes multiple physical and psychosocial challenges to people surviving a stroke. Stroke is a broad term, which includes ischaemic stroke, stroke caused by intracerebral or subarachnoid haemorrhage, or cerebral venous thrombosis, and stroke of an unspecified type (Sacco et al., 2013 \& Feigin, 2013).

According to Ostwald (2015), fifteen million people globally are affected by stroke and sixty percent of them either die shortly after the stroke or are permanently disabled as a result of stroke. It is projected that the global stroke burden would increase from 38 million of disability-adjusted life years in 1990 to 61 million in 2020 (Ostwald, 2015).

Stroke is associated with profound negative impacts on survivors' physical, psychological and social functioning (Carod-Artal, 2009). Stroke patients experienced different physical challenges in daily life after discharge from hospital. Common challenges reported included decreased range of motion and strength of limbs; difficulties in mobility and maintaining balance; sensory impairment in upper or lower limbs, impaired vision; eating or swallowing difficulties; and aphasia and/or dysphasia (Koenig-Bruhin et al., 2013). 
Furthermore, stroke survivors may have experienced boundaries in activity of daily living (ADL) well before the stroke and decline in ADL independence at the time of stroke, and these may have continued for weeks or months afterwards (Capistrant, Wang, Liu, \& Glymour, 2013).

Stroke patients meet several physical, psychological and social challenges consequential to stroke. To cope with these challenges, stroke patients frequently need to perform physical and functional training, which often require rhythmic practices and hard work. Other aspects of post-stroke care include various self-monitoring practices and health-promoting behaviors such as blood pressure monitoring and control, smoking cessation, healthy diet, and monitoring for signs and symptoms of recurrent stroke (Jauch et al., 2013 \& National Stroke Foundation, 2011).

Management of their psychological and social challenges requires reorientation of stroke patients' own perceptions of post-stroke changes, and increased self-awareness and determination to learn to new roles after stroke (White et al., 2012). All these illustrate attention to the integral role of stroke patients themselves in self-managing their health.

Knowledge and skills to resolve daily health troubles and make health decisions, and capabilities to locate resources and understand health information to promote stroke recovery are also of top priority. For example, they need to problem-solve the barriers to participating in activities and exercise; and to set goals related to their exercise regimens for pacing themselves during the recovery period (Peoples et al., 2011). The ability to recognize problems associated with stroke, and adapts their behaviors and attitudes using self-management skills such as problem-solving or decision-making were significant factors for reintegrating into the community after stroke. Obviously, the core self-management skills are essential in long-term management of stroke, particularly when stroke patients have to depend on themselves after they have returned home from hospital (Woodman et al., 2014). Eftekhar et al., (2010) defined stroke discharge planning as "an integrated and synchronized utilization of medical, social, educational, and occupational plans and measures for retraining stroke patients in order to optimize physical, psychological, social, and vocational potential, participation with physiologic and environmental restrictions". It is an active process beginning in hospitalization care and has a goal of returning to the community and aims at assisting the patients to recapture their functional independence and to return to an active and productive lifestyle.

Nurses are playing an important role in discharge planning. They have a most important planning responsibility for each patient. In general, nurses can offer nutritional education, assist with meal plans, behavior change and construction confidence and flexibility among stroke patients to better cope with their condition. This responsibility involves providing information to stroke survivors and their families and relatives and get in touch with different aspects in the patient care, in acute care, rehabilitation clinics, primary care, and municipalities (Maslakpak \& Shams, 2015).

\subsection{Significance of the Study}

Stroke was the second leading cause of global death after ischaemic heart disease between 2000 and 2012. It accounted for 6.7 million (11.9\%) global deaths in 2012 (World Health Organization (WHO), 2016a). However, the death toll attributable to stroke remains high and it is estimated that it will increase to 7.8 million deaths worldwide by 2030 (WHO, 2016b). Worldwide stroke-related illness, disability and early death are set to double in next 15 years by 2035. The incidence of stroke in Egypt is 270 thousand yearly; about 75 thousands of them are left disabled (Egyptian Ministry of Health, 2017).

Stroke is associated with deep unconstructive impacts on patients' physical, psychological and social functioning. Some of the most important impacts are limitations in physical mobility, activities and functions, and loss of independence. Moreover, stroke patients found difficulties in participating in their pre-stroke social roles, work or leisure activities. In addition to post-stroke depression, several studies have reported that stroke survivors had a higher risk of developing psychological distress, anxiety or lowered levels of self-esteem (White, Barrientos \& Dunn, 2014).

As soon as the stroke patients are discharged from hospital, they are expected to learn to manage their physical and functional disabilities, and emotional disturbance consequential to the stroke. They are also expected to adapt to the limitations after stroke and reintegrate into the community to resume their pre-stroke roles and lives, or to assume new family and/or social roles. Also it is hoped that findings of this study might help in improving quality of patient care and activity of daily living to establish evidence based data that can promote nursing practice and research. 


\subsection{Aim of the Study}

This study aimed to evaluate the effect of a design discharge planning program for stroke patients on their quality of life and activity of daily living through:

1- Assessing needs based on knowledge of patients with stroke.

2- Assessing quality of life and activity of daily living among patients with stroke.

3- Developing and implementing a design discharge planning program for patients with stroke.

4- Evaluating the effect of a design discharge planning program on stroke patients' quality of life and activity of daily living.

\subsection{Research Hypothesis}

H1-The mean knowledge scores of patients with stroke who are exposed to a design discharge planning program will be higher than before.

H2- The mean quality of life scores of patients with stroke who are exposed to a design discharge planning program will be higher than before.

H3-The mean activity of daily living scores of patients with stroke who are exposed to a design discharge planning program will be higher than before.

H4- There will be a positive relation between patient's knowledge, quality of life, activity of daily living and with their socio demographic characteristics post implementing a design discharge planning program.

\section{Subjects and Methods}

\subsection{Research Design}

A quasi experimental design was utilized to fulfill the aim of this study.

\subsection{Setting of the Study}

The study was conducted at the stroke department and stroke outpatient clinics affiliated to Benha University Hospital which is located at Al-Qualubia Governorate.

\subsection{Study Subjects}

Convenient (50) sample of patients with stroke who are meeting:

Inclusion criteria: adult male and female, with confirmed diagnosis of stroke, did not having any educational program, and able to communicate.

Exclusion criteria: Patients with vasculitis, thrombophilic diseases, infectious vasculopathy, radiation induced vasculopathy, sickle cell disease, dementia and aphasia were excluded from the study, Patients with Transient Ischemic Attack(TIA) without progression to stroke as well as those with severe heart, liver or renal disease that may considerably influence the Quality of Life (QOL) were also excluded.

\subsection{Tools for Data Collection: To Achieve the Aim of the Present Study Three Tools Were Used}

Tool (1): Structured Interviewing questionnaire for patients: which was developed in a simple clear Arabic language by the researchers based on literature review (Ziki, 2017 and Ostwald, 2015). It included the following parts:

Part 1. Patients'Socio-demographicData:It developed by the researchers and included; age, sex, level of education, occupation, residence and marital status.

Part 2. Medical History. It was developed by the researchers, constructed and reviewed utilizing the most recent and relevant literature Ziki, (2017), it consists of series of questions to elicit patients past medical history. It is composed of (6) questions which include previous admission to hospital, onset of stroke occurrence, history of any previous neurological disease, causes of stroke, type of clot suffered by patient, disability resulting from stroke and any psychological disorder.

Part 3. Patients' Knowledge Assessment: Prepared by the researchers after reviewing of the related literature (Ziki, 2017 and Ostwald, 2015). It was used to assess patient level of knowledge. It divided into two parts:

I-Part one: included series of questions to elicit patient knowledge related to stroke, it is composed of (15) questions about stroke, which included: definition, causes, types, signs \& symptoms, diagnosis, complications, prevention and treatments.

II-Part two: which consisted of (8) questions about patients activities of daily living. It is composed of 
knowledge related to skin care, bathing, oral care, criteria for wearing clothes, healthy food, feeding, movement aids and exercises.

Scoring system: Patient knowledge assessment sheet consists of closed ended questions. Multiple choice questions, the answers were evaluated using model key answer prepared by the researcher. The score was "zero "for "incorrect" answer, and "1" for correct answer, the scores of the items were summed-up and the total divided by the number of the items, giving a mean score for the part. These scores were converted into a percent score, and means and standard deviations were computed, Total knowledge scores were (23), considered as:

- $\quad$ Satisfactory if score $>60 \%$ of the maximum score.

- Unsatisfactory if score $\leq 60 \%$ of the minimum score.

Tool (2): Stroke Specific Quality of Life Scale (SS-QOL):

It was adopted from Williams et al., (1999). The internal consistency and reliability were examined using Cronbach's alpha (recommended value $\alpha \geq 0.78$ ) (Muus, Williams \& Ringsberg, 2007 and Boosman et al, 2010).

The SS-QOL, which is a disease-specific QOL measure, consists of 49 items encompassing 12 domains, which include energy (three questions), family role (three questions), language (five questions), mobility (six questions), mood (five questions), personality (three questions), self-care (five questions), the social role (five questions), thinking (three questions), upper extremity function (five questions), vision (three questions), and work/productivity (three questions). Each item is ranked on a five-point Likert scale in which level one means completely agreed while level five means completely disagree. The summary score of this scale is an un-weighted average of the 12 domains.

\section{Scoring system:}

Scoring of the SS-QOL is rated on a 5-point Likert scale. Response options are scored as 5 ("no help needed/no trouble at all/strongly disagree"), 4 ("a little help/a little trouble/moderately disagree"), 3 ("some help/some trouble/neither agree nor disagree"), 2 ("a lot of help/a lot of trouble/moderately agree"), and 1 ("total help/could not do it at all/strongly agree"). The SS-QOL provides domain scores and a summary score, with higher scores indicating better function. The total score ranges from 49 to 245, with higher scores indicating a better QOL.

Tool (3): Barthel Index for Activities of Daily Living (ADL):

The Barthel scale is an ordinal scale used to measure performance in activities of daily living (ADL). Each performance item is rated on this scale with a given number of points assigned to each level or ranking. It uses ten variables describing ADL and mobility. A higher number is associated with a greater likelihood of being able to live at home with a degree of independence following discharge from hospital. The amount of time and physical assistance required to perform each item are used in determining the assigned value of each item.

The Barthel index has demonstrated test-retest reliability (0.89) as well as high correlations (0.74-0.8) with other measures of physical disability (Sullivan et al., 2007).

\section{Tools validity and reliability:}

All tools of the current study were reviewed by five experts, four professors in the field of medical surgical nursing and one assistant professor in medicine to ensure its clarity and applicability. The tools were modified according to the experts' opinion on simplicity of the sentences and suitability of the content. The reliability was done by Cronbach's Alpha coefficient test for tool $1(0.846)$. While, the reliability was already done by Cronbach's Alpha coefficient test for tool $2(\geq 0.78)$ and tool 3 ( 0.89) .

\section{Pilot study:}

It was conduct on $10 \%$ of total patients' sample in order to test simplicity and applicability of the study tools as well as estimation of time needed to fill in the tools. Required modifications were done in the form of adding or omission of some questions. Patients involved in pilot study were excluded from the main study samples.

\section{Ethical considerations:}

The present study was conducted under the approval of the Faculty of Nursing Ethical Committee, Benha University. An official permission to conduct the study was obtained by the researcher from the head of the stroke unit at Benha University Hospital.

Oral agreement for voluntary participation was attained after explaining the aim and objectives of the study to patients included in the study, and assured maintaining anonymity and confidentiality of subjective data through coding of all data. Patients were also informed that they are allowed to choose to participate or not in the study 
and to withdraw from it at any time without giving any reasons.

Field work:

The study was carried out through four phases: Assessment, planning, implementation, and evaluation.

Assessment phase:

This includes review of the current and past available literature and theoretical knowledge was done using books, internet, and magazines to develop the tools for data collection.

- At initial interview the researchers introduce themselves to begin now of contact, explain the purpose of a design discharge planning program and fill out the structured interview sheet (tool 1) to assess patient's knowledge pre implementation of a design discharge planning program and the researchers fill out Stroke Specific Quality of Life Scale(tool 2), and Barthel Index for Activities of Daily Living(Tool 3) to assess patient's activity of daily living pre implementation of discharge planning program.

Planning phase: General objective: The general objective of developing a design discharge planning program was to improve knowledge, quality of life and activity of daily living practice of stroke patients.

Specific objectives: By the end of the developing a design discharge planning program, stroke patients should be able to:

- Define the meaning of stroke.

- Enumerate causes and associated risk factors of stroke.

- Illustrate the signs and symptoms of stroke.

- Identify the required investigations and methods of treatment of stroke.

- Discuss how to improve quality of care for stroke patient.

- Explain activity of daily living that can be participated by stroke patient.

Implementation phase:

- Data collection for this study was carried out in the period from November 2018 to November 2019.

- The aim of the study was explained by the researchers to all patients included in the study and took written consent from patients who were able to read and write and oral consent from illiterate patients.

- In addition to the allocated assessment times before and after implementing a design discharge planning program. After that, the socio-demographic and medical data sheet was completed for all patients of. A design discharge planning program was utilized for the study group subjects by the researchers who used on daily bases from time of admission till patient discharge.

- The tools filled through interviewing

- The researchers visited the study setting 3 days per week from 10 am to $12 \mathrm{pm}$ and sometimes from 12.00p.m. To 3.00p.m., each patient was interviewed individually. The purpose of the study was explained to the patients prior to answer the questions. The study was carried out at morning, and after noon shifts.

- The discharge planning program was conducted through 4 sessions. The duration of each session ranged from 45-60 minutes. The first sessions started by theoretical part about knowledge related to definition of stroke, its signs and symptoms, risk factors, types of medication used as treatment, complications and prevention the recurrent attack.

- The second session about knowledge related to activity of daily living.

- The third session concerning with instructions for patients about movement, elimination, eating and drinking, bathing, clothing, medications and assistive devices and healthy life style.

- And the fourth session about some exercises the patient can perform to regain his physical health.

- Each session was started by a summary about what has been discussed in the previous one and presenting the objectives of the new session, using simple Arabic language, also the session ended by a summary of its content and feedback from the patient was obtained to ensure that they got the maximum benefit. The teaching materials used were lecturer, discussion, each patient was received the illustrated booklet as a hand out, an open channel of communication was achieved between the researcher and patients to assure understanding, answer any question and to verify information.

- Patients were informed about the time of the next session at the end of the session. 
- $\quad$ A design discharge planning program: It was developed by researchers from literature reviews, researcher experience and opinions of the medical and nursing expertise based on patient assessment needs to maintain health promotion for patients. The teaching booklet was revised and modified based on the expertise comments; it was written in Arabic using simple language with illustration. It consists of knowledge of the patient related to stroke (definition, causes, types, risk factors, prevention) and pre discharge instructions for patients about activities of daily living and improving quality of life.

Evaluation phase:

- The effect of a design discharge planning program on patient's knowledge, quality of life and activity of daily living was reached, through comparing level of patient's knowledge using tool (1) part 3, it included three phases (immediate post program to evaluate their gain of knowledge and then 1st and 3rd month after implementing the discharge planning program as a follow up assessment . Also, quality of life and activity of daily living has been evaluated $\left(1^{\text {st }}\right.$ and $3^{\text {rd }}$ month after program implementation) by the researchers through filling the study tool (2) and tool (3).

Limitation of the study:

- One limitation of the study is that we took only 3 measurements over the 3-month period. To better quantify the changes in the Barthel Index score after stroke, more measurements are recommended during the follow-up partly because the improvements are extended until 12 months post-stroke.

- There were exclusion criteria which limited the sample size and this may limit the generalizability of the results. Also the result can't be generalized because the sample was collected from one hospital only.

\section{Statistical Analysis}

The collected data were organized, categorized, analyzed using SPSS 11.0 statistical software packages. Data were presented using descriptive statistics in the form of frequencies and percentages for qualitative variables, and mean and standard deviations for quantitative variables. Correlation coefficient, T- test for comparison of means and Chi- square test was used for comparing frequency between studied groups. Statistical significance was considered at P- value $<0.05$.

\section{Results}

Table 1 revealed that most of the studied patients (80\%) within age group of (50-60) years old within (mean age 54.22 and 6.22 SD). Sixty eight percent of them were married .Regarding their educational level, more than half of them were illiterate and less than half of them $(46 \%)$ were workers. As regards residence, it was found that the majority of studied patients $(92 \%)$ were resided in rural areas.

Table 2 demonstrates that, around two thirds $(68 \%)$ of studied patients were having stroke due to ischemic causes, without a history of smoking $(60 \%)$. Also, Table 2 revealed that more than half of the studied patients $(56 \%$ \& $52 \%$,respectively) had a previous stroke hospitalization, history with onset of stroke ranged from $1-10$ days respectively .Concerning risk factors for stroke, it was found that less than half (48\%) of studied patients were having hypertension. In relation to disability from stroke, $(48 \%)$ of the studied patients were have two or more disabilities like sensory, motor, mental or speech.

Table 3 revealed that the highest mean score preprogram implementation was $(1.88 \pm 0.84)$ regarding studied patient knowledge related to activities of daily living ,but the lowest mean score was $(0.20 \pm 0.40)$ related to definition of stroke. On the other hand, the mean scores were increased immediate, 1 st and 3rd month post program implementation to $(3.72 \pm 1.132,0.92 \pm 0.27,0.94 \pm 0.23),(2.82 \pm 1.107,0.84 \pm 0.36,0.68 \pm 0.46)$ and $(2.72 \pm 1.11,0.74 \pm 0.43,0.52 \pm 0.49)$ respectively for knowledge related activities of daily living, healthy food and radiographic examination. With a highly statistical significant difference between studied patients knowledge pre and post program implementation were observed at $p$ - values of $\leq 0.001$ although, the mean scores got low among phases of follow up.

Figure 1 Documented an unsatisfactory knowledge level among $86 \%$ of the studied patients pre design discharge planning program implementation. However, immediately after design discharge planning program implementation, only $(26 \%)$ of the studied patients got an unsatisfactory knowledge level. The knowledge level increased to (74\%) immediately after planning program implementation with slight decrease to $44 \%$ \& $30 \%$ at follow up periods $\left(1^{\text {st }}\right.$ and $3^{\text {rd }}$ month) after planning program implementation.

Table 4 Illustrates mean score and standard deviation of studied patients related to barthel index scale pre and post program implementation. This table revealed that total mean scores of barthel index categories were increased and improved post program implementation than preprogram implementation, where was $(53.2 \pm$ 
22.71) preprogram and increased to $(71 \pm 18.05)$ post $1^{\text {st }}$ month and improved to $(79.5 \pm 17.21)$ post $3^{\text {rd }}$ month with a highly statistical significant difference between studied patients barthel index categories pre and post program implementation were observed at $\mathrm{p}$ - values of $\leq 0.001$.

Table 5 shows comparison between the studied patients regarding their total percentage score of Barthel index Scale pre and post program implementation. It revealed that approximately two thirds $(60 \%)$ of studied patients were severely dependent preprogram implementation which decreased to $(40 \%)$ post $1^{\text {st }}$ month and $(22 \%)$ post 3 rd month. On other hand (16\%) of studied patients were mild dependent preprogram implementation which was increased to $(28 \%$ and $52 \%)$ post program implementation $\left(1^{\text {st }}\right.$ and $3^{\text {rd }}$ month $)$ respectively. There is a highly statistical significant difference between studied patients pre and post program implementation were observed at $\mathrm{p}-$ values of $\leq 0.001$.

Table 6 demonstrated that preprogram implementation, the lowest mean scores $(9.18 \pm 1.85,9.42 \pm 2.01,9.46 \pm$ $0.963)$ respectively related to vision, work/productivity, and energy and a highest mean scores were (12.98 \pm $4.465,11.6 \pm 3.622$ ) related to mobility and language respectively. While post program implementation ( $\left(1^{\text {st }}\right.$ month) general improvement in mean scores items of quality of life $(20.02 \pm 3.962,19.02 \pm 3.396,17.72 \pm$ 3.954 and $17.6 \pm 3.815)$ related to mobility, language, mood and social roles, respectively but decreased to $(16.36 \pm 5.65,15.48 \pm 5.02,14.32 \pm 5.31$ and $13.5 \pm 4.63)$ respectively A statistical significant difference between studied patients pre and post program implementation were observed at $p$ - values of $\leq 0.001$.

Table 7 Total percentage score of quality of life pre and post program implementation. This table shows an improvement in quality of life for patient while preprogram implementation only $(10 \%)$ had good QoL) reached to $(38 \%$ and $24 \%)$ post $1^{\text {st }}$ and $3^{\text {rd }}$ month respectively. There was statistically significant difference between them pre and post program implementation was observed on $p$ value $\leq 0.001$.

Table 8 Relation between socio-demographic and knowledge pre and post program .This table shows a general improvement in Patients knowledge post program implementation as compared to preprogram implementation. but specifically as seen from this table the university, worked, female, reside in urban area obtained a high mean scores in different periods of program implementation. There was statistically significant difference between them pre and post program implementation was observed on $p$ value $\leq 0.001$.

Table 9 Relation between socio demographic and Quality of life pre and post program implementation .This table shows a general improvement in patients' quality of life post program implementation (as compared to preprogram implementation. But specifically as seen from this table the university, single, worked, female and reside in urban area obtained a high mean scores in different periods of program implementation. There was statistically significant difference between them pre and post program implementation was observed on $p$ value $\leq 0.001$.

Table 10 Relation between socio demographic and Barthel index pre and post program implementation .This table shows a general improvement in Patients barthel index scale post program implementation as compared to preprogram implementation. But specifically as seen from this table the university, worked, female, single and reside in urban area obtained a high mean scores in different periods of program implementation. There was statistically significant difference between them pre and post program implementation was observed on $p$ value $\leq 0.001$.

Table 11 Correlation coefficient related to age, knowledge, quality of life, barthel index pre and post program implementation. Negative correlations were observed as regards age with knowledge, quality of life, barthel index. Which reflect the younger people have a highest knowledge, quality of life and barthel index scores than older people? On other hand appositive correlation was observed between knowledge, quality of life, barthel index, as well as between quality of life and barthel index along pre and post program implementation. Highly statistically significant differences were observed at $\mathrm{p} \leq 0.001$. 
Table 1. Frequency and percentage distribution of the studied patients related to their socio -demographic characteristics $(\mathrm{N}=50)$.

\begin{tabular}{|c|c|c|c|}
\hline \multirow{2}{*}{$\begin{array}{l}\text { Socio demographic } \\
\text { characteristics }\end{array}$} & \multirow[t]{2}{*}{ Items } & \multicolumn{2}{|c|}{ Studied patients $(\mathrm{n}=50)$} \\
\hline & & $\mathrm{N}$ & $\%$ \\
\hline & $-35-<40$ & 2 & 4.0 \\
\hline \multirow[t]{2}{*}{ Age } & $-40-<50$ & 8 & 16.0 \\
\hline & $-50-65$ & 40 & 80.0 \\
\hline \multirow[t]{2}{*}{$\mathrm{X} \pm \mathrm{SD}$} & & \pm 6 . & \\
\hline & -Male & 24 & 48.0 \\
\hline \multirow[t]{2}{*}{ Gender } & -Female & 26 & 52.0 \\
\hline & -Illiterate & 28 & 56.0 \\
\hline \multirow[t]{4}{*}{ Level of education } & -Primary & 10 & 20.0 \\
\hline & -Secondary & 8 & 16.0 \\
\hline & -University & 4 & 8.0 \\
\hline & -Not work & 2 & 4.0 \\
\hline \multirow[t]{4}{*}{ Occupation } & -Work & 23 & 46.0 \\
\hline & -House wife & 16 & 32.0 \\
\hline & -Retirement & 9 & 18.0 \\
\hline & -Single & 4 & 8.0 \\
\hline \multirow[t]{3}{*}{ Marital status } & -Married & 34 & 68.0 \\
\hline & -Divorced & 9 & 18.0 \\
\hline & -Widow & 3 & 6.0 \\
\hline \multirow[t]{2}{*}{ Residence } & -Rural & 46 & 92.0 \\
\hline & -Urban & 4 & 8.0 \\
\hline
\end{tabular}

Table 2. Frequency and percentage distribution of the studied patients according to their medical history $(\mathrm{N}=50)$

\begin{tabular}{cccc}
\hline Medical history & Items & \multicolumn{2}{c}{ Studied patients (n=50) } \\
\cline { 2 - 4 } & & $\mathrm{N}$ & $\%$ \\
\hline $\begin{array}{c}\text { Previous hospitalization } \\
\text { with stroke }\end{array}$ & Yes & 28 & 56.0 \\
\hline Onset of stroke & No & 22 & 44.0 \\
\hline Nervousness history & $-1-10$ day & 8 & 52.0 \\
& $-11-20$ day & 16 & 16.0 \\
Smoking history & -more than 20 day & 22 & 32.0 \\
\hline Risk factors for stroke & Yes & 24 & 44.0 \\
& No & 20 & 48.0 \\
& Yes & 30 & 60.0 \\
\hline Etiology of stroke & No & 8 & 16.0 \\
\hline
\end{tabular}




\begin{tabular}{cccc}
\hline & -Ischemic & 34 & 68.0 \\
\hline & -Sensory & 8 & 16.0 \\
& -Mobility & 2 & 4.0 \\
Disability from stroke & -Mental & 4 & 8.0 \\
& -Speech & 8 & 16.0 \\
& -Orientation state & 4 & 8.0 \\
& -Two or more disabilities & 24 & 48.0 \\
\hline
\end{tabular}

Table 3. Mean score and standard deviation of studied patients related to their knowledge about stroke pre and post program implementation $(\mathrm{N}=50)$

\begin{tabular}{|c|c|c|c|c|c|c|c|}
\hline \multirow{2}{*}{$\begin{array}{c}\text { Patient } \\
\text { knowledge } \\
\text { about stroke }\end{array}$} & \multirow{2}{*}{$\begin{array}{l}\text { Before } \\
\text { implement } \\
\text { ation }\end{array}$} & \multicolumn{3}{|c|}{ Post implementation } & \multirow[t]{2}{*}{ P1 } & \multirow[t]{2}{*}{ P2 } & \multirow[t]{2}{*}{$\mathrm{P} 3$} \\
\hline & & Immediate & $1^{\text {st }}$ month & $3^{\text {rd }}$ month & & & \\
\hline $\begin{array}{l}\text { Stroke } \\
\text { definition } \\
\bar{X} \pm \mathrm{SD}\end{array}$ & $0.20 \pm 0.40$ & $0.58 \pm 0.49$ & $0.46 \pm 0.49$ & $0.42 \pm 0.49$ & $<0.001^{* * *}$ & $<0.01^{* *}$ & $<0.05^{*}$ \\
\hline T test & \multicolumn{2}{|c|}{$\mathrm{T} 1=4.25$} & $\mathrm{~T} 2=2.9$ & $\mathrm{~T} 3=2.46$ & & & \\
\hline $\begin{array}{c}\text { Predisposing } \\
\text { factors } \\
\bar{X} \pm \mathrm{SD}\end{array}$ & $0.22 \pm 0.41$ & $0.54 \pm 0.49$ & $\begin{array}{c}0.48 \\
\pm 0.499\end{array}$ & $0.46 \pm 0.49$ & $<0.001^{* * *}$ & $<0.01^{* *}$ & $<0.01^{* *}$ \\
\hline T test & $\mathrm{T} 1=3$ & .5416 & $\mathrm{~T} 2=2.846$ & $\mathrm{~T} 3=2.656$ & & & \\
\hline $\begin{array}{c}\text { Types of stroke } \\
\bar{X} \pm \mathrm{SD}\end{array}$ & $0.30 \pm 0.458$ & $0.9 \pm 0.3$ & $0.78 \pm 0.41$ & $0.64 \pm 0.48$ & $<0.001^{* * *}$ & $<0.001^{* * *}$ & $<0.001^{* * *}$ \\
\hline $\mathrm{T}$ test & $\mathrm{T} 1=$ & 7.749 & $\mathrm{~T} 2=5.521$ & $\mathrm{~T} 3=3.623$ & & & \\
\hline $\begin{array}{c}\text { Transient } \\
\text { attack } \\
\bar{X} \pm \mathrm{SD}\end{array}$ & $0.28 \pm 0.44$ & $0.56 \pm 0.49$ & $0.52 \pm 0.499$ & $0.5 \pm 0.5$ & $<0.01^{* *}$ & $<0.05^{*}$ & $<0.05^{*}$ \\
\hline T test & $\mathrm{T} 1=$ & .0064 & $\mathrm{~T} 2=2.551$ & $\mathrm{~T} 3=2.335$ & & & \\
\hline $\begin{array}{l}\text { Ischemic } \\
\text { stroke } \\
\bar{X} \pm \mathrm{SD}\end{array}$ & $0.24 \pm 0.42$ & $0.52 \pm 0.49$ & $0.5 \pm 0.5$ & $0.48 \pm 0.49$ & $<0.01^{* *}$ & $<0.01^{* *}$ & $<0.01^{* *}$ \\
\hline T test & $\mathrm{T} 1=$ & 3.068 & $\mathrm{~T} 2=2.818$ & $\mathrm{~T} 3=2.63$ & & & \\
\hline $\begin{array}{c}\text { Hemorrhagic } \\
\text { stroke } \\
\bar{X} \pm \mathrm{SD}\end{array}$ & $0.34 \pm 0.47$ & $0.7 \pm 0.45$ & $0.64 \pm 0.48$ & $0.62 \pm 0.48$ & $<0.001^{* * *}$ & $<0.01^{* *}$ & $<0.01^{* *}$ \\
\hline T test & $\mathrm{T} 1=$ & 3.93 & $\mathrm{~T} 2=3.157$ & $\mathrm{~T} 3=2.94$ & & & \\
\hline $\begin{array}{c}\text { Signs and } \\
\text { symptoms of } \\
\text { stroke } \\
\bar{X} \pm \mathrm{SD}\end{array}$ & $0.34 \pm 0.47$ & $0.78 \pm 0.41$ & $0.72 \pm 0.44$ & $0.6 \pm 0.48$ & $<0.001^{* * *}$ & $<0.001^{* * *}$ & $<0.01^{* *}$ \\
\hline T test & $\mathrm{T} 1=$ & 4.99 & $\mathrm{~T} 2=4.17$ & $\mathrm{~T} 3=2.736$ & & & \\
\hline $\begin{array}{l}\text { Complications } \\
\text { of stroke } \\
\bar{X} \pm \mathrm{SD}\end{array}$ & $0.42 \pm 0.49$ & $0.82 \pm 0.38$ & $0.76 \pm 0.42$ & $0.62 \pm 0.48$ & $<0.001^{* * *}$ & $<0.001^{* * *}$ & $<0.05^{*}$ \\
\hline
\end{tabular}




\begin{tabular}{|c|c|c|c|c|c|c|c|}
\hline T test & \multicolumn{2}{|c|}{$\mathrm{T} 1=4.56$} & $\mathrm{~T} 2=3.72$ & $\mathrm{~T} 3=2.06$ & & & \\
\hline $\begin{array}{c}\text { Prevention of } \\
\text { stroke } \\
\bar{X} \pm \mathrm{SD}\end{array}$ & $0.46 \pm 0.49$ & $0.74 \pm 0.43$ & $0.74 \pm 0.43$ & $0.7 \pm 0.45$ & $<0.001^{* * *}$ & $<0.01^{* *}$ & $<0.05^{*}$ \\
\hline $\mathrm{T}$ test & $\mathrm{T} 1=$ & 5.81 & $\mathrm{~T} 2=3.037$ & $\mathrm{~T} 3=2.551$ & & & \\
\hline $\begin{array}{c}\text { Healthy food } \\
\bar{X} \pm \mathrm{SD}\end{array}$ & $0.48 \pm 0.499$ & $0.92 \pm 0.27$ & $0.84 \pm 0.36$ & $0.74 \pm 0.43$ & $<0.001^{* * *}$ & $<0.001^{* * *}$ & $<0.01^{* *}$ \\
\hline $\mathrm{T}$ test & $\mathrm{T} 1=$ & 6531 & $\mathrm{~T} 2=4.18$ & $\mathrm{~T} 3=2.82$ & & & \\
\hline $\begin{array}{c}\text { Healthy oils } \\
\bar{X} \pm \mathrm{SD}\end{array}$ & $0.52 \pm 0.49$ & $0.9 \pm 0.3$ & $0.8 \pm 0.4$ & $0.78 \pm 0.41$ & $<0.001^{* * *}$ & $<0.01^{* *}$ & $<0.01^{* *}$ \\
\hline T test & $\mathrm{T} 1=$ & 4.67 & $\mathrm{~T} 2=3.13$ & $\mathrm{~T} 3=2.877$ & & & \\
\hline $\begin{array}{c}\text { Healthy juices } \\
\bar{X} \pm \mathrm{SD}\end{array}$ & $0.34 \pm 0.47$ & $0.88 \pm 0.32$ & $0.78 \pm 0.41$ & $0.66 \pm 0.47$ & $<0.001^{* * *}$ & $<0.001^{* * *}$ & $<0.01^{* *}$ \\
\hline $\mathrm{T}$ test & $\mathrm{T} 1=$ & 6.71 & $\mathrm{~T} 2=4.988$ & $\mathrm{~T} 3=3.40$ & & & \\
\hline
\end{tabular}

Cont. Table 3

\begin{tabular}{|c|c|c|c|c|c|c|c|}
\hline \multirow{2}{*}{$\begin{array}{c}\text { Patient } \\
\text { knowledge } \\
\text { about stroke }\end{array}$} & \multirow{2}{*}{$\begin{array}{c}\text { Before } \\
\text { implementation }\end{array}$} & \multicolumn{3}{|c|}{ Post implementation } & \multirow[t]{2}{*}{$\mathrm{P} 1$} & \multirow[t]{2}{*}{$\mathrm{P} 2$} & \multirow[t]{2}{*}{ P3 } \\
\hline & & Immediate & $1^{\text {st }}$ month & $3^{\text {rd }}$ month & & & \\
\hline $\begin{array}{c}\text { Radiographic } \\
\text { examination } \\
\bar{X} \pm \mathrm{SD}\end{array}$ & $0.32 \pm 0.46$ & $0.94 \pm 0.23$ & $0.68 \pm 0.46$ & $0.52 \pm 0.49$ & $<0.001^{* * *}$ & $<0.001^{* * *}$ & $<0.05^{*}$ \\
\hline $\mathrm{T}$ test & \multicolumn{2}{|c|}{$\mathrm{T} 1=7.82$} & $\mathrm{~T} 2=3.91$ & $\mathrm{~T} 3=2.10$ & & & \\
\hline $\begin{array}{c}\text { Laboratory } \\
\text { examination } \\
\bar{X} \pm \mathrm{SD}\end{array}$ & $0.42 \pm 0.493$ & $0.86 \pm 0.34$ & $0.82 \pm 0.38$ & $0.68 \pm 0.46$ & $<0.001^{* * *}$ & $<0.001^{* * *}$ & $<0.01^{* *}$ \\
\hline T test & \multicolumn{2}{|c|}{$\mathrm{T} 1=5.21$} & $\mathrm{~T} 2=4.56$ & $\mathrm{~T} 3=2.73$ & & & \\
\hline $\begin{array}{c}\text { Methods of } \\
\text { treatment } \\
\bar{X} \pm \mathrm{SD}\end{array}$ & $0.5 \pm 0.5$ & $0.94 \pm 0.23$ & $0.8 \pm 0.4$ & $0.74 \pm 0.43$ & $<0.001^{* * *}$ & $<0.01^{* *}$ & $<0.05^{*}$ \\
\hline T test & \multicolumn{2}{|c|}{$\mathrm{T} 1=5.65$} & $\mathrm{~T} 2=3.31$ & $\mathrm{~T} 3=2.57$ & & & \\
\hline $\begin{array}{l}\text { Knowledge } \\
\text { related to } \\
\text { activities of } \\
\text { daily living } \\
\quad \bar{X} \pm \mathrm{SD}\end{array}$ & $1.88 \pm 0.84$ & $3.72 \pm 1.132$ & $2.82 \pm 1.107$ & $2.72 \pm 1.114$ & $<0.001^{* * *}$ & $<0.001^{* * *}$ & $<0.001^{* * *}$ \\
\hline T test & \multicolumn{2}{|c|}{$\mathrm{T} 1=9.24$} & $\mathrm{~T} 2=4.80$ & $\mathrm{~T} 3=4.257$ & & & \\
\hline $\begin{array}{c}\text { Total } \\
\text { knowledge } \\
\text { scores } \\
\bar{X} \pm \mathrm{SD}\end{array}$ & $7.56 \pm 3.353$ & $15.46 \pm 2.78$ & $13.08 \pm 2.92$ & $10.66 \pm 3.31$ & $<0.001^{* * *}$ & $<0.001^{* * *}$ & $<0.001^{* * *}$ \\
\hline T test & $\mathrm{T} 1=12$ & & $\mathrm{~T} 2=9.73$ & $\mathrm{~T} 3=4.65$ & & & \\
\hline
\end{tabular}

(*) Statistically significant at $\mathrm{p} \leq 0.05(* *)$ Highly statistically significant at $\mathrm{p} \leq 0.01$.

$(* * *)$ Very highly statistically significant at $\mathrm{p} \leq 0.001$ 


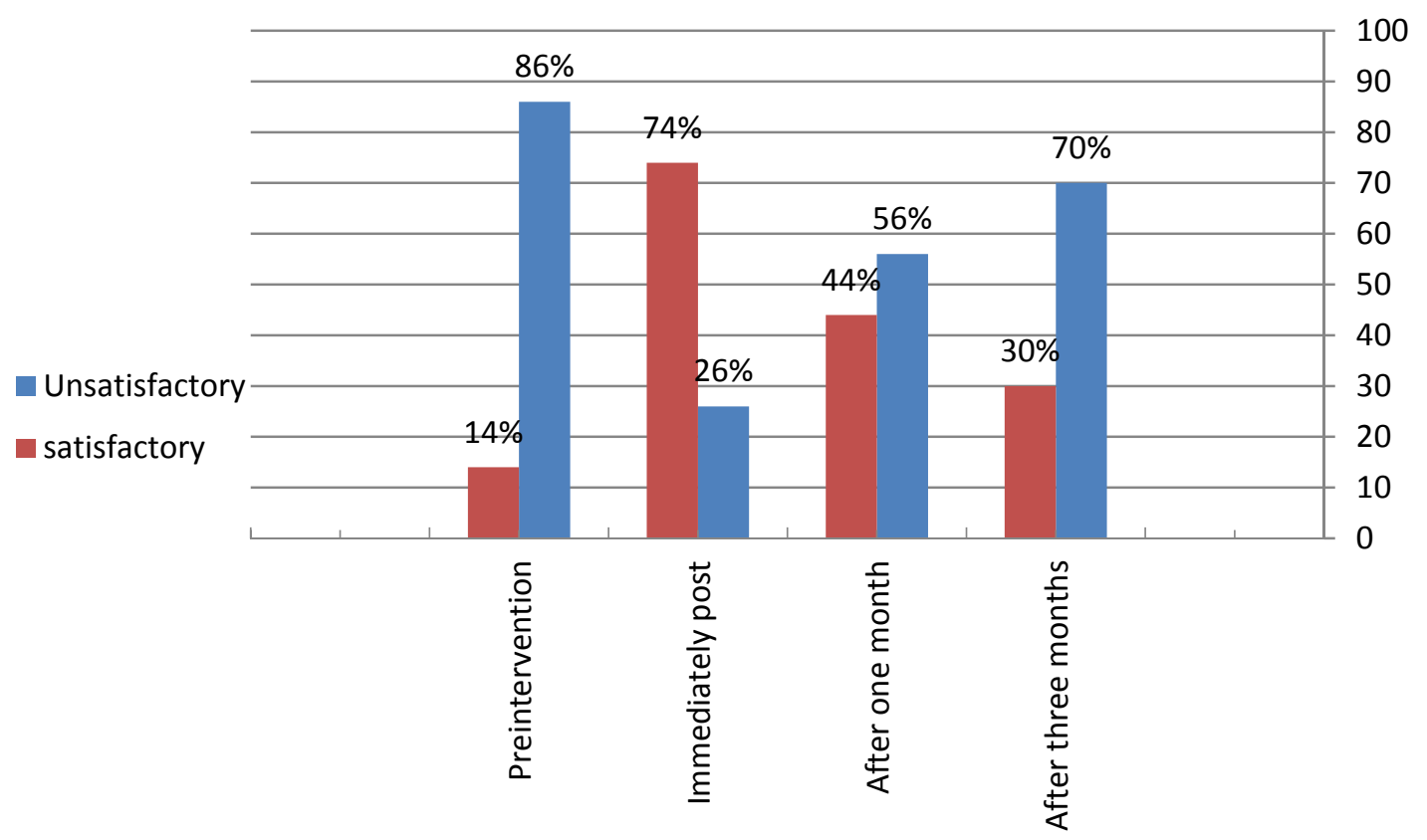

Figure 1. Total knowledge percentage scores of the studied subjects all through assessment periods $(\mathrm{N}=50)$

Table 4. Mean score and standard deviation of studied patients related to Barthel index scale pre and post program implementation $(\mathrm{N}=50)$

\begin{tabular}{|c|c|c|c|c|c|c|c|c|c|c|}
\hline \multirow[t]{2}{*}{ Items } & \multicolumn{2}{|c|}{ Pre program } & \multicolumn{2}{|c|}{$\begin{array}{l}\text { Post program } \\
\text { (after one } \\
\text { month) }\end{array}$} & \multirow[t]{2}{*}{ T-test } & \multirow[t]{2}{*}{ P-value } & \multicolumn{2}{|c|}{$\begin{array}{l}\text { Post program } \\
\text { (after } 3 \\
\text { months) }\end{array}$} & \multirow[t]{2}{*}{ T-test } & \multirow[t]{2}{*}{ P-value } \\
\hline & $X$ & SD & $X$ & SD & & & $X$ & SD & & \\
\hline Feeding & 4.3 & 2.83 & 6 & 3 & 2.91 & $<0.01^{* *}$ & 7.7 & 3.49 & 5.3506 & $<0.001^{* * *}$ \\
\hline Bathing & 2 & 2.44 & 3.9 & 2.07 & 4.198 & $<0.001^{* * *}$ & 4.3 & 1.73 & 5.4373 & $<0.001^{* * *}$ \\
\hline Grooming & 2.4 & 2.49 & 3.8 & 2.13 & 3.021 & $<0.01^{* *}$ & 4.4 & 1.624 & 4.7572 & $<0.001^{* * *}$ \\
\hline Dressing & 5.6 & 3.26 & 7.1 & 3.17 & 2.33 & $<0.05^{*}$ & 8.6 & 2.83 & 4.9139 & $<0.001^{* * *}$ \\
\hline Bowels & 5.2 & 2.99 & 7.2 & 2.48 & 3.640 & $<0.001^{* * *}$ & 8.1 & 3.3 & 4.6049 & $<0.001^{* * *}$ \\
\hline Bladder & 5.8 & 3.05 & 7.1 & 2.84 & 2.206 & $<0.05^{*}$ & 8.6 & 2.83 & 4.7586 & $<0.001^{* * *}$ \\
\hline Toilet use & 6.2 & 2.74 & 8.48 & 2.47 & 4.370 & $<0.001^{* * *}$ & 9 & 2.23 & 4.37 & $<0.001^{* * *}$ \\
\hline $\begin{array}{c}\text { Transfers } \\
\text { (Bed to } \\
\text { chair and } \\
\text { back) }\end{array}$ & 7.1 & 3.88 & 9.5 & 3.64 & 3.189 & $<0.01^{* *}$ & 10.2 & 3.73 & 3.189 & $<0.01^{* *}$ \\
\hline Mobility & 7.4 & 3.90 & 9.8 & 2.82 & 3.52 & $<0.001^{* * *}$ & 10.6 & 3.82 & 4.1449 & $<0.001^{* * *}$ \\
\hline Stairs & 5.3 & 3.22 & 7.3 & 2.86 & 3.283 & $<0.01^{* *}$ & 8.6 & 2.65 & 5.5955 & $<0.001^{* * *}$ \\
\hline Total & 53.2 & 22.71 & 71 & 18.05 & 4.338 & $<0.001^{* * *}$ & 79.5 & 17.21 & 6.5265 & $<0.001^{* * *}$ \\
\hline
\end{tabular}

(*) Statistically significant at $\mathrm{p} \leq 0.05$

(**) Highly statistically significant at $\mathrm{p} \leq 0.01$

(***) Very highly statistically significant at $\mathrm{p} \leq 0.001$ 
Table 5. Comparison between the studied patients regarding their total percentage score of Barthel index Scale pre and post program implementation (after one and three months $(\mathrm{N}=50)$

\begin{tabular}{|c|c|c|c|c|c|c|c|c|c|c|c|}
\hline \multirow{2}{*}{$\begin{array}{l}\text { Total } \\
\text { Percentage } \\
\text { Classification }\end{array}$} & \multirow[t]{2}{*}{$\begin{array}{l}\text { Classification } \\
\text { meaning }\end{array}$} & \multicolumn{2}{|c|}{$\begin{array}{l}\text { Pre program } \\
\text { implementation }\end{array}$} & \multicolumn{2}{|c|}{$\begin{array}{l}\text { Post program } \\
\text { implementation } \\
\text { (after one month) }\end{array}$} & \multirow[t]{2}{*}{$\mathrm{X} 2$} & \multirow[t]{2}{*}{ P-value } & \multicolumn{2}{|c|}{$\begin{array}{l}\text { Post program } \\
\text { implementation } \\
\text { (after } 3 \text { month }\end{array}$} & \multirow[t]{2}{*}{$\mathrm{X} 2$} & \multirow[t]{2}{*}{ P-value } \\
\hline & & $\mathrm{N}$ & $\%$ & $\mathrm{~N}$ & $\%$ & & & $\mathrm{~N}$ & $\%$ & & \\
\hline $20-60 \%$ & $\begin{array}{l}\text { severely } \\
\text { dependent }\end{array}$ & 30 & 60.0 & 20 & 40.0 & & & 11 & 22.0 & & \multirow{4}{*}{$0.0088^{* * *}$} \\
\hline $65-85 \%$ & $\begin{array}{l}\text { moderately } \\
\text { dependent }\end{array}$ & 12 & 24.0 & 16 & 32.0 & & & 13 & 26.0 & & \\
\hline $90-95 \%$ & $\begin{array}{l}\text { mildly } \\
\text { dependent }\end{array}$ & 8 & 16.0 & 14 & 28.0 & & & 26 & 52.0 & & \\
\hline $100 \%$ & $\begin{array}{l}\text { completely } \\
\text { independent }\end{array}$ & 0 & 0.0 & 0 & 0.0 & 8.415 & $0.0148^{*}$ & 0 & 0.0 & 9.4686 & \\
\hline
\end{tabular}

(*) Statistically significant at $\mathrm{p} \leq 0.05$

$(* * *)$ Very highly statistically significant at $\mathrm{p} \leq 0.001$

Table 6. Mean score and standard deviation of studied patients related to quality of life scale pre and post program implementation $(\mathrm{N}=50)$

\begin{tabular}{|c|c|c|c|c|c|c|c|c|c|c|}
\hline \multirow[t]{2}{*}{ Items } & \multicolumn{2}{|c|}{ Pre program } & \multicolumn{2}{|c|}{$\begin{array}{c}\text { Post program } \\
\text { (after one month) }\end{array}$} & \multirow[t]{2}{*}{ T-test } & \multirow[t]{2}{*}{$\mathrm{P}$-value } & \multicolumn{2}{|c|}{$\begin{array}{l}\text { Post program } \\
\text { (after } 3 \text { month) }\end{array}$} & \multirow[t]{2}{*}{ T-test } & \multirow[t]{2}{*}{ P-value } \\
\hline & $\mathrm{X}$ & SD & $\mathrm{X}$ & SD & & & $\mathrm{X}$ & SD & & \\
\hline Energy & 9.46 & 0.963 & 12.16 & 1.901 & 8.96 & $<0.001^{* * *}$ & 10.56 & 1.68 & 4.019 & $<0.001^{* * *}$ \\
\hline Family Roles & 9.88 & 1.829 & 11.86 & 1.587 & 5.78 & $<0.001^{* * *}$ & 10.66 & 1.98 & 2.05 & $<0.05^{*}$ \\
\hline Language & 11.6 & 3.623 & 19.02 & 3.396 & 10.579 & $<0.001^{* * *}$ & 15.48 & 5.02 & 4.433 & $<0.001^{* * *}$ \\
\hline Mobility & 12.98 & 4.465 & 20.02 & 3.962 & 8.34 & $<0.001^{* * *}$ & 16.36 & 5.65 & 3.320 & $<0.01^{* *}$ \\
\hline Mood & 10.76 & 3.547 & 17.72 & 3.954 & 9.28 & $<0.001^{* * *}$ & 14.32 & 5.31 & 3.944 & $<0.001^{* * *}$ \\
\hline Personality & 9.74 & 1.338 & 11.52 & 1.043 & 7.45 & $<0.001^{* * *}$ & 10.76 & 1.40 & 3.735 & $<0.001^{* * *}$ \\
\hline Self Care & 10.76 & 3.707 & 17.34 & 3.937 & 8.62 & $<0.001^{* * *}$ & 13.34 & 4.66 & 3.06 & $<0.01^{* *}$ \\
\hline Social Roles & 10.9 & 3.634 & 17.6 & 3.815 & 9.00 & $<0.001^{* * *}$ & 13.5 & 4.63 & 3.125 & $<0.01^{* *}$ \\
\hline Thinking & 9.6 & 1.637 & 11.68 & 1.173 & 7.33 & $<0.001^{* * *}$ & 10.76 & 1.69 & 3.49 & $<0.001^{* * *}$ \\
\hline $\begin{array}{l}\text { Upper Extremity } \\
\text { Function }\end{array}$ & 10.92 & 3.520 & 17.58 & 4.414 & 8.34 & $<0.001^{* * *}$ & 14.4 & 5.36 & 3.83 & $<0.001^{* * *}$ \\
\hline Vision & 9.18 & 1.85 & 11.68 & 1.223 & 7.97 & $<0.001^{* * *}$ & 10.12 & 2.05 & 2.407 & $<0.05^{*}$ \\
\hline Work/Productivity & 9.42 & 2.01 & 11.64 & 1.144 & 6.79 & $<0.001^{* * *}$ & 10.34 & 2.01 & 2.29 & $<0.05^{*}$ \\
\hline Total Quality of life & 125.2 & 29.88 & 179.72 & 29.666 & 9.156 & $<0.001^{* * *}$ & 150.44 & 39.70 & 3.59 & $<0.001^{* * *}$ \\
\hline
\end{tabular}

(*) Statistically significant at $\mathrm{p} \leq 0.05(* *)$ Highly statistically significant at $\mathrm{p} \leq 0.01$

$(* * *)$ Very highly statistically significant at $\mathrm{p} \leq 0.001$ 
Table 7. Total percentage score of quality of life among studied patients Pre and Post Program Implementation $(\mathrm{N}=50)$

\begin{tabular}{|c|c|c|c|c|c|c|c|c|c|c|c|}
\hline \multirow{3}{*}{$\begin{array}{c}\text { Total Percentage } \\
\text { Classification }\end{array}$} & \multicolumn{11}{|c|}{ Quality of life } \\
\hline & \multirow[t]{2}{*}{$\begin{array}{c}\text { Classification } \\
\text { meaning }\end{array}$} & \multicolumn{2}{|c|}{$\begin{array}{l}\text { Pre program } \\
\text { implementation }\end{array}$} & \multicolumn{2}{|c|}{$\begin{array}{l}\text { Post (after } \\
\text { one month) }\end{array}$} & \multirow[t]{2}{*}{$\mathrm{X} 2$} & \multirow[t]{2}{*}{ P-value } & \multicolumn{2}{|c|}{$\begin{array}{c}\text { Post } \\
\text { (after } 3 \text { month) }\end{array}$} & \multirow[t]{2}{*}{$\mathrm{X} 2$} & \multirow[t]{2}{*}{ P-value } \\
\hline & & $\mathrm{N}$ & $\%$ & $\mathrm{~N}$ & $\%$ & & & $\mathrm{~N}$ & $\%$ & & \\
\hline $\begin{array}{l}\text { Less than } 49 \\
\text { score }(<20 \%)\end{array}$ & $\begin{array}{l}\text { Very poor } \\
\text { quality }\end{array}$ & 0 & 0.0 & 0 & 0.0 & \multirow{5}{*}{21.6695} & \multirow{5}{*}{$0.00002^{* *}$} & 0 & 0.0 & & \multirow{5}{*}{$0.00358^{* *}$} \\
\hline $49-<147(<60 \%)$ & Poor quality & 38 & 76.0 & 15 & 30.0 & & & 27 & 54 & 11.266 & \\
\hline $147-196(<80 \%)$ & Fair quality & 7 & 14.0 & 16 & 32.0 & & & 11 & 22 & & \\
\hline $\begin{array}{c}196-<245 \\
(<100 \%)\end{array}$ & Good quality & 5 & 10.0 & 19 & 38.0 & & & 12 & 24 & & \\
\hline $245(100 \%)$ & Perfect quality & 0 & 0.0 & 0 & 0.0 & & & 0 & 0.0 & & \\
\hline
\end{tabular}

$(* *)$ Highly statistically significant at $\mathrm{p} \leq 0.01(* * *)$ Very highly statistically significant at $\mathrm{p} \leq 0.001$

Table 8 . Relation between socio-demographic and knowledge pre and post program $(\mathrm{N}=50)$

\begin{tabular}{|c|c|c|c|c|c|c|c|c|c|c|c|c|c|c|}
\hline \multirow{5}{*}{$\begin{array}{l}\begin{array}{l}\text { Socio } \\
\text { demo- }\end{array} \\
\text { graphic } \\
\text { Chara- } \\
\text { cteristics }\end{array}$} & \multicolumn{14}{|c|}{ Knowledge } \\
\hline & \multirow{3}{*}{\multicolumn{2}{|c|}{$\begin{array}{l}\text { Pre } \\
\text { implementation }\end{array}$}} & \multirow{3}{*}{\multicolumn{2}{|c|}{$\begin{array}{l}\text { Immediately } \\
\text { post } \\
\text { implementatio } \\
\mathrm{n}\end{array}$}} & \multirow[t]{4}{*}{$\mathrm{T}$ test } & \multirow[t]{4}{*}{ p-value } & \multirow{3}{*}{\multicolumn{2}{|c|}{$\begin{array}{l}\text { Post } \\
\text { implementation } \\
\text { (after one month) }\end{array}$}} & \multirow[t]{4}{*}{$\mathrm{T}$ test } & \multirow[t]{4}{*}{ p-value } & \multirow{3}{*}{\multicolumn{2}{|c|}{$\begin{array}{l}\text { Post } \\
\text { implementatio } \\
\mathrm{n} \quad \text { (after } 3 \\
\text { month) }\end{array}$}} & \multirow[t]{4}{*}{$\mathrm{T}$ test } & \multirow[t]{4}{*}{ p-value } \\
\hline & & & & & & & & & & & & & & \\
\hline & & & & & & & & & & & & & & \\
\hline & $\mathrm{X}$ & SD & $\mathrm{X}$ & $\mathrm{SD}$ & & & $\mathrm{X}$ & $\mathrm{SD}$ & & & $\mathrm{X}$ & $\mathrm{SD}$ & & \\
\hline \multicolumn{15}{|l|}{ Gender } \\
\hline Male & 8.62 & 3.351 & 14.8 & 3.21 & 9.46 & $<0.001^{* * *}$ & 12.79 & 3.34 & 4.32 & $<0.001^{* * *}$ & 10.42 & & & \\
\hline (24) & & & & & & & & & & & & 3.66 & 1.77 & $<0.05^{*}$ \\
\hline $\begin{array}{l}\text { Female } \\
(26)\end{array}$ & 8.81 & 2.842 & 16.04 & 2.17 & 14.28 & $<0.001^{* * *}$ & 13.35 & 2.45 & 6.18 & $<0.001^{* * *}$ & 10.88 & 2.94 & 2.600 & $<0.05^{*}$ \\
\hline \multicolumn{15}{|c|}{ Level of education } \\
\hline $\begin{array}{l}\text { Illiterate } \\
\text { (28) }\end{array}$ & 7.07 & 1.709 & 13.71 & 2.03 & 17.68 & $<0.001^{* * *}$ & 11.178 & 1.98 & 8.30 & $<0.001^{* * *}$ & 8.43 & 1.72 & 2.96 & $<0.01^{* *}$ \\
\hline $\begin{array}{l}\text { Primary } \\
\text { (10) }\end{array}$ & 8.2 & 0.75 & 16 & 0.774 & 51.24 & $<0.001^{* * *}$ & 14 & 1.34 & 11.93 & $<0.001^{* * *}$ & 12 & 2.28 & 5.00 & $\begin{array}{l}<0.001 \\
* * *\end{array}$ \\
\hline $\begin{array}{l}\text { Secondary } \\
(8)\end{array}$ & 11.75 & 2.634 & 18.5 & 1.12 & 16.68 & $<0.001^{* * *}$ & 16 & 1.32 & 4.07 & $<0.001^{* * *}$ & 13.87 & 2.15 & 1.76 & $\begin{array}{l}0.098 \\
\text { n.s }\end{array}$ \\
\hline $\begin{array}{l}\text { University } \\
\text { (4) }\end{array}$ & 15.5 & 0.5 & 20.25 & 0.83 & 34.694 & $<0.001^{* * *}$ & 18.25 & 0.43 & 8.31 & $<0.001^{* * *}$ & 16.5 & 0.5 & 2.82 & $<0.05^{*}$ \\
\hline \multicolumn{15}{|c|}{ Occupation } \\
\hline $\begin{array}{l}\text { Not work } \\
\text { (2) }\end{array}$ & 7.5 & 0.5 & 15.5 & 0.5 & 80.00 & $<0.001^{* * *}$ & 14.5 & 0.5 & 14.00 & $<0.01^{* *}$ & 10.5 & 0.5 & 6.00 & $<0.05^{*}$ \\
\hline $\begin{array}{l}\text { Work } \\
\text { (23) }\end{array}$ & 10.78 & 3.189 & 17.17 & 2.55 & 11.073 & $<0.001^{* * *}$ & 15.04 & 2.51 & 5.037 & $<0.001^{* * *}$ & 12.87 & 2.98 & 2.29 & $<0.05^{*}$ \\
\hline
\end{tabular}




\begin{tabular}{|c|c|c|c|c|c|c|c|c|c|c|c|c|c|c|}
\hline $\begin{array}{l}\text { House } \\
\text { wife (16) }\end{array}$ & 7.5 & 1.541 & 15.06 & 1.477 & 25.05 & $<0.001^{* * *}$ & 12.19 & 1.70 & 8.16 & $<0.001^{* * *}$ & 9.69 & 2.17 & 3.285 & $<0.01^{* *}$ \\
\hline $\begin{array}{l}\text { Retirement } \\
\text { (9) }\end{array}$ & 5.889 & 1.099 & 11.78 & 1.133 & 26.38 & $<0.001^{* * *}$ & 9.33 & 0.66 & 8.040 & $<0.001^{* * *}$ & & 0.78 & 1.97 & $<0.05^{*}$ \\
\hline \multicolumn{15}{|c|}{ Marital status } \\
\hline $\begin{array}{l}\text { Single } \\
\text { (4) }\end{array}$ & 15 & 0.707 & 19.75 & 0.433 & 40.51 & $<0.001^{* * *}$ & 17.25 & 0.83 & 4.13 & $<0.01^{* *}$ & 15.75 & 0.83 & 1.37 & $\begin{array}{l}0.217 \\
\text { n.s }\end{array}$ \\
\hline $\begin{array}{l}\text { Married } \\
(34)\end{array}$ & 7.97 & 1.617 & 15.09 & 2.318 & 11.34 & $<0.001^{* * *}$ & 12.85 & 2.38 & 9.90 & $<0.001^{* * *}$ & 10.26 & 2.71 & 4.23 & $\begin{array}{l}<0.001 \\
* * *\end{array}$ \\
\hline $\begin{array}{l}\text { Divorced } \\
\text { (3) }\end{array}$ & $\begin{array}{l}15.66 \\
7\end{array}$ & 0.471 & 20.33 & 0.943 & 31.295 & $<0.001^{* * *}$ & 18.33 & 0.47 & 6.92 & $<0.01^{* *}$ & 16.67 & 0.47 & 2.599 & $\begin{array}{l}0.06 \\
\text { n.s }\end{array}$ \\
\hline $\begin{array}{l}\text { Widow } \\
\text { (9) }\end{array}$ & 6.444 & 1.499 & 13.33 & 1.155 & 25.29 & $<0.001^{* * *}$ & 10.33 & 1.05 & 5.28 & $<0.001^{* * *}$ & 7.88 & 0.99 & 0.592 & $\begin{array}{l}0.562 \\
\text { n.s }\end{array}$ \\
\hline \multicolumn{15}{|l|}{ Residence } \\
\hline $\begin{array}{l}\text { Rural } \\
\text { (46) }\end{array}$ & 8.17 & 2.58 & 15.11 & 2.623 & 9.86 & $<0.001^{* * *}$ & 12.72 & 2.75 & 8.17 & $<0.001^{* * *}$ & 10.22 & 3.07 & 3.46 & $\begin{array}{l}<0.001 \\
* * *\end{array}$ \\
\hline Urban (4) & 15 & 0.707 & 19.5 & 0.5 & 36.74 & $<0.001^{* * *}$ & 17.25 & 0.83 & 4.13 & $<0.01^{* *}$ & 15.75 & 0.83 & 1.37 & $\begin{array}{l}0.218 \\
\text { n.s }\end{array}$ \\
\hline
\end{tabular}

n.s :not statistically significant at p-value 0.05

$\left(^{* *}\right)$ Highly statistically significant at $\mathrm{p} \leq 0.01$ $\left(^{*}\right)$ Statistically significant at $\mathrm{p} \leq 0.05$

$\left(^{* * *}\right)$ Very highly statistically significant at $\mathrm{p} \leq 0.001$

Table 9. Relation between socio demographic and Quality of life pre and post program Implementation $(\mathrm{N}=50)$

\begin{tabular}{|c|c|c|c|c|c|c|c|c|c|c|}
\hline \multirow{4}{*}{$\begin{array}{c}\text { Socio } \\
\text { demographic } \\
\text { Characteristics }\end{array}$} & \multicolumn{10}{|c|}{ Quality of life } \\
\hline & \multirow{2}{*}{\multicolumn{2}{|c|}{$\begin{array}{c}\text { Pre } \\
\text { implementation }\end{array}$}} & \multirow{2}{*}{\multicolumn{2}{|c|}{$\begin{array}{c}\text { Post } \\
\text { implementation } \\
\text { (after one month) }\end{array}$}} & \multirow{3}{*}{$\mathrm{T}$ test } & \multirow{3}{*}{ p-value } & \multirow{2}{*}{\multicolumn{2}{|c|}{$\begin{array}{c}\text { Post } \\
\text { implementation } \\
\text { (after three } \\
\text { month) }\end{array}$}} & \multirow[t]{3}{*}{$\mathrm{T}$ test } & \multirow[t]{3}{*}{$\mathrm{p}$-value } \\
\hline & & & & & & & & & & \\
\hline & $X$ & SD & $X$ & SD & & & $X$ & SD & & \\
\hline \multicolumn{11}{|c|}{ Gender } \\
\hline Male (24) & 123.62 & 35.46 & 170.70 & 33.99 & 6.7767 & $<0.001^{* * *}$ & 151.91 & 44.968 & 2.4196 & $<0.05^{*}$ \\
\hline Female (26) & 126.65 & 23.48 & 188.03 & 21.93 & 13.5045 & $<0.001^{* * *}$ & 149.076 & 34.083 & 2.762 & $<0.01^{* *}$ \\
\hline \multicolumn{11}{|c|}{ Level of education } \\
\hline Illiterate (28) & 109.67 & 17.13 & 162.35 & 28.65 & 11.1552 & $<0.001^{* * *}$ & 124.893 & 28.80 & 2.402 & $<0.05^{*}$ \\
\hline Primary (10) & 122.8 & 2.85 & 198.2 & 5.50 & 85.9087 & $<0.001^{* * *}$ & 166 & 27.864 & 4.877 & $<0.001^{* * *}$ \\
\hline $\begin{array}{c}\text { Secondary } \\
(8)\end{array}$ & 146.12 & 24.57 & 199.12 & 7.11 & 14.6496 & $<0.001^{* * *}$ & 193.5 & 9.420 & 5.09 & $<0.001^{* * *}$ \\
\hline $\begin{array}{c}\text { University } \\
\text { (4) }\end{array}$ & 198 & 0.70 & 216.25 & 4.81 & 26.5151 & $<0.001^{* * *}$ & 204.25 & 2.487 & 0.834 & $<0.01^{* *}$ \\
\hline
\end{tabular}

\begin{tabular}{ccccccccccc}
\hline \multicolumn{10}{c}{ Occupation } \\
\hline Not work (2) & 119 & 3 & 196 & 4 & 108.894 & $<0.001^{* * *}$ & 181 & 1 & \\
\hline Work (23) & 143.60 & 32.86 & 196 & 20.82 & 85.9087 & $<0.001^{* * *}$ & 179.478 & 32.119 & 27.727 & $<0.001^{* * *}$ \\
\hline $\begin{array}{c}\text { House wife } \\
(16)\end{array}$ & 117.31 & 9.217 & 179.87 & 23.17 & 17.7353 & $<0.001^{* * *}$ & 131.125 & 22.042 & 3.744 & $<0.001^{* * *}$ \\
\hline
\end{tabular}




\begin{tabular}{ccccccccccc}
\hline $\begin{array}{c}\text { Retirement } \\
(9)\end{array}$ & 93.55 & 7.81 & 134.22 & 4.021 & 32.7043 & $<0.001^{* * *}$ & 103.778 & 7.495 & 2.831 & $<0.05^{*}$ \\
\hline \multicolumn{7}{c}{ Marital status } \\
\hline Single (4) & 172.75 & 24.26 & 207 & 6.51 & 9.6397 & $<0.001^{* * *}$ & 201.25 & 4.850 & 2.304 & $<0.05^{*}$ \\
\hline Married (34) & 118 & 18.47 & 180.79 & 27.25 & 13.4856 & $<0.001^{* * *}$ & 149.176 & 37.514 & 4.347 & $<0.001^{* * *}$ \\
\hline Divorced(3) & 198 & 0.816 & 215.66 & 5.43 & 22.7263 & $<0.001^{* * *}$ & 203 & 1.414 & 5.305 & $<0.001^{* * *}$ \\
\hline Widow (9) & 107 & 10.50 & 151.55 & 22.26 & 12.7958 & $<0.001^{* * *}$ & 115.111 & 10.608 & 1.629 & $0.1227 \mathrm{n} . \mathrm{s}$ \\
\hline & & & & & & & & & \\
\hline Rural (46) & 120 & 24.39 & 177.19 & 29.56 & 10.5512 & $<0.001^{* * *}$ & 145.978 & 38.2 & 3.887 & $<0.001^{* * *}$ \\
\hline Urban (4) & 185 & 20.79 & 208.75 & 5.53 & 7.8032 & $<0.001^{* * *}$ & 201.75 & 4.0233 & 1.581 & 0.1648 \\
\hline
\end{tabular}

n.s :not statistically significant at $\mathrm{p}$-value $0.05\left(^{*}\right)$ Statistically significant at $\mathrm{p} \leq 0.05$

$\left(^{* *}\right)$ Highly statistically significant at $\mathrm{p} \leq 0.01\left(^{* * *}\right)$ Very highly statistically significant at $\mathrm{p} \leq 0.001$

Table 10. Relation between socio demographic and Barthel index pre and post program implementation $(\mathrm{N}=50)$

\begin{tabular}{|c|c|c|c|c|c|c|c|c|c|c|}
\hline \multirow{3}{*}{$\begin{array}{l}\text { Socio demographic } \\
\text { Items }\end{array}$} & \multicolumn{10}{|c|}{ Barthel index } \\
\hline & \multicolumn{2}{|c|}{ Pre program } & \multicolumn{2}{|c|}{$\begin{array}{l}\text { Post program } \\
\text { (after one month) }\end{array}$} & \multirow[t]{2}{*}{ T-test } & \multirow[t]{2}{*}{$\mathrm{P}$-value } & \multicolumn{2}{|c|}{$\begin{array}{l}\text { Post program } \\
\text { (after } 3 \text { month) }\end{array}$} & \multirow[t]{2}{*}{ T-test } & \multirow[t]{2}{*}{ P-value } \\
\hline & $\mathrm{X}$ & SD & $\mathrm{X}$ & SD & & & $\mathrm{X}$ & SD & & \\
\hline Gender & 51.875 & 25.405 & 59.80 & 26.08 & 1.0664 & 0.2918 & 71.667 & 18.408 & 4.461 & $<0.001^{* * *}$ \\
\hline -Male $(n=24)$ & & & & & & n.s & & & & \\
\hline -Female $(n=26)$ & 54.423 & 19.823 & 66.92 & 17.87 & 2.388 & $<0.05^{*}$ & 86.730 & 12.164 & 9.822 & $<0.001^{* * *}$ \\
\hline $\begin{array}{l}\text { Level of education } \\
\text {-Illiterate }(\mathrm{n}=28)\end{array}$ & 40.536 & 14.036 & 49.643 & 15.69 & 2.288 & $<0.05^{*}$ & 72.857 & 16.059 & 10.715 & $<0.001^{* * *}$ \\
\hline- Primary $(n=10)$ & 61.50 & 13.610 & 79.50 & 10.356 & 3.328 & $<0.001^{* * *}$ & 92.50 & 2.50 & 15.841 & $<0.001^{* * *}$ \\
\hline -Secondary $(n=8)$ & 68.75 & 27.128 & 77.50 & 23.452 & 0.690 & $0.5014 \mathrm{n} . \mathrm{s}$ & 80 & 22.079 & 2.274 & $<0.05^{*}$ \\
\hline -University $(\mathrm{n}=4)$ & 91.25 & 2.165 & 90.25 & 2.165 & 0.6532 & $0.5378 \mathrm{n} . \mathrm{s}$ & 92.50 & 2.50 & 2.673 & $<0.01^{* *}$ \\
\hline $\begin{array}{l}\text { Occupation } \\
- \text { Not work }(n=2)\end{array}$ & 67.50 & 2.50 & 71.60 & 2.50 & 1.6400 & $0.2427 \mathrm{n} . \mathrm{s}$ & 72.50 & 2.50 & 10.00 & $<0.001^{* * *}$ \\
\hline -Work $(n=23)$ & 67.608 & 21.962 & 76.304 & 18.95 & 0.0503 & $0.9601 \mathrm{n} . \mathrm{s}$ & 85.652 & 14.766 & 4.821 & $<0.001^{* * *}$ \\
\hline -House wife $(\mathrm{n}=16)$ & 46.563 & 11.418 & 61.56 & 15.48 & 3.1186 & $<0.01^{* *}$ & 86.875 & 7.262 & 21.065 & $<0.001^{* * *}$ \\
\hline -Retirement(n=9) & 25.556 & 4.969 & 32.222 & 4.157 & 3.087 & $<0.01^{* *}$ & 52.222 & 6.713 & 22.576 & $<0.001^{* * *}$ \\
\hline $\begin{array}{l}\text { Marital status } \\
\text {-Single }(\mathrm{n}=4)\end{array}$ & 92.50 & 2.50 & 91.25 & 2.165 & 0.756 & $0.4783 \mathrm{n} . \mathrm{s}$ & 93.75 & 2.165 & 2.673 & $<0.01^{* *}$ \\
\hline -Married(n=34) & 50.735 & 18.516 & 63.235 & 21.174 & 2.591 & $<0.05^{*}$ & 78.676 & 18.837 & 7.48 & $<0.001^{* * *}$ \\
\hline
\end{tabular}


Cont. table 10

\begin{tabular}{|c|c|c|c|c|c|c|c|c|c|c|}
\hline \multirow{3}{*}{$\begin{array}{l}\begin{array}{l}\text { Socio } \\
\text { demographic }\end{array} \\
\text { Items }\end{array}$} & \multicolumn{10}{|c|}{ Barthel index } \\
\hline & \multicolumn{2}{|c|}{ Pre program } & \multicolumn{2}{|c|}{$\begin{array}{l}\text { Post program } \\
\text { (after one month) }\end{array}$} & \multirow[t]{2}{*}{ T-test } & \multirow[t]{2}{*}{ P-value } & \multicolumn{2}{|c|}{$\begin{array}{l}\text { Post program } \\
\text { (after } 3 \text { month) }\end{array}$} & \multirow[t]{2}{*}{ T-test } & \multirow[t]{2}{*}{ P-value } \\
\hline & $\mathrm{X}$ & SD & $X$ & SD & & & $\mathrm{X}$ & SD & & \\
\hline $\begin{array}{l}\text {-Divorced } \\
(n=3)\end{array}$ & 90.68 & 2.38 & 91.667 & 2.357 & 0.5104 & $\begin{array}{l}0.6367 \mathrm{n} . \\
\mathrm{s}\end{array}$ & 93.333 & 2.357 & 12.98 & $<0.001^{* * *}$ \\
\hline $\begin{array}{l}\text {-Widow } \\
(\mathrm{n}=9)\end{array}$ & 33.333 & 7.453 & 42.222 & 6.713 & 2.658 & $<0.05^{*}$ & 72.222 & 10.304 & 21.62 & $<0.001^{* * *}$ \\
\hline $\begin{array}{l}\text { Residence } \\
\text {-Rural } \\
(\mathrm{n}=46)\end{array}$ & 50 & 20.799 & 60.87 & 21.627 & 2.457 & $<0.05^{*}$ & 78.369 & 17.478 & 7.38 & $<0.001^{* * *}$ \\
\hline$-\operatorname{Urban}(\mathrm{n}=4)$ & 90.68 & 2.38 & 91.25 & 2.165 & 0.3547 & $0.735 \mathrm{n} . \mathrm{s}$ & 92.5 & 2.5 & 2.67 & $<0.01^{* *}$ \\
\hline
\end{tabular}

Table 11. Correlation coefficient related to age, knowledge, quality of life, barthel index pre and post program implementation $(\mathrm{N}=50)$

\begin{tabular}{|c|c|c|}
\hline r-p value & $\mathrm{r}$ & $\mathrm{P}$-value \\
\hline \multicolumn{3}{|l|}{ Age with knowledge } \\
\hline Pre & -0.78749 & $<.00001^{* * *}$ \\
\hline Immediately post & -0.77887 & $<.00001^{* * *}$ \\
\hline After one month & -0.724358 & $<.00001^{* * *}$ \\
\hline After three months & -0.7065126 & $<.00001^{* * *}$ \\
\hline \multicolumn{3}{|c|}{ Age with quality of life } \\
\hline Pre & -0.73258 & $<.00001^{* * *}$ \\
\hline Post(after one month) & -0.65547 & $<.00001^{* * *}$ \\
\hline After three months & -0.68283 & $<.00001^{* * *}$ \\
\hline \multicolumn{3}{|c|}{ Age with Barthel index } \\
\hline Pre & -0.68376 & $<.00001^{* * *}$ \\
\hline Post(after one month) & -0.61134 & $0.000446^{*}$ \\
\hline After three months & -0.47812 & $<.00001^{* * *}$ \\
\hline \multicolumn{3}{|c|}{ Knowledge with quality of life } \\
\hline Pre & 0.88211 & $<.00001^{* * *}$ \\
\hline After one month & 0.874110 & $<.00001^{* * *}$ \\
\hline After three months & 0.88850 & $<.00001^{* * *}$ \\
\hline \multicolumn{3}{|c|}{ Knowledge with Barthel index } \\
\hline Pre & 0.763680 & $<.00001^{* * *}$ \\
\hline After one month & 0.888059 & $<.00001^{* * *}$ \\
\hline After three months & 0.590049 & $<.00001^{* * *}$ \\
\hline \multicolumn{3}{|c|}{ Quality of life with Barthel index } \\
\hline Pre & 0.83853 & $<.00001^{* * *}$ \\
\hline Post(after one month) & 0.6718 & $<.00001^{* * *}$ \\
\hline After three months & 0.68204 & $<.00001^{* * *}$ \\
\hline
\end{tabular}

$\left({ }^{* * *}\right)$ Very highly statistically significant at $\mathrm{p} \leq 0.001$ 


\section{Discussion}

This study aimed to evaluate the effect of a design discharge plan for stroke patients on their quality of life and activity of daily living.

Regarding age, the present study revealed that most of the studied patients were in the age category $50-60$ years old within mean age of $(54.22 \pm 6.22 \mathrm{SD})$. This may be due to the fact that physiological and health life changes in older adults make them the most vulnerable group for chronic diseases which may be a risk for stroke.

This result agreed with the study conducted by Zaky et al., (2015), who was studying "Strategies of Daily Living Rehabilitative Activities for Post Stroke Patients at Minia University Hospital" and found that most of the study group were within age category $>51-60$ years old within mean (52.03 and 15.04 SD).

Also this result in accordance with Serda et al., (2015) who was studying "determining of life quality and associated factors in stroke patients" and Kamel et al., (2010) who studied about "health related quality of life in stroke survivors measured by the stroke impact scale "who found that in their studies the patient with stroke were in mean age group $(55.40 \pm 12.50 \mathrm{SD}),(53.3 \pm 8.2 \mathrm{SD})$ respectively.

This is in line with Kothari et al. (1997) studied about "Patient's awareness of stroke signs, symptoms and risk factors" and Pancioli et al. (1998) studied about "Public perception of stroke warning signs and knowledge of potential risk factors" who they pointed that cardiovascular and metabolic disease incidence increase with age, people who more likely to experience strokes are old people. Age is the single most important risk factor for stroke. The stroke rate increase more than doubles in both men and women for each successive 10 years after age 55 years old.

As regard to patients' gender, the present study revealed that more than half of studied patients were females. From the researcher point of view this may be due to the females expose to stressors during their personal life ,or due to hormonal fluctuation, changes that happened in different stages of their life such pregnancy ,childbirth ,feeding and menopause.

This result agreed with Zaky et al., (2015) who stated that more than half of his sample were female and also this result supported by Seana, et al., (2012) about "sex differences in long-term outcomes after stroke functional outcomes, handicap, and quality of life" who recently reported that females had more severe strokes, higher short term mortality in comparison to males. This is incongruent with Khedr et al., (2013) who reported in his study about "epidemiological study and risk factors of stroke in Assiut governorate, Egypt: Health-based study "that prevalence rate of stroke is high in men than woman.

Also this result revealed that more than two thirds of sample was married and half of them were illiterate. From the researcher point of view, it may be due to the married people were more accountable to stroke than single people which it may be related to social and psychological constant worry in their life and their families responsibility and educational opportunities are more in the urban than in the rural areas.

These results supported by Serda et al., (2015) and Zaky et al, (2015), who found that most of his sample were married and two third of them were illiterate. Also agreed with Khedr et al., (2013) who found there was significantly high occurrence of stroke in illiterate patients.

As regard to residence, most of the sample was lived in rural areas, this result agreed with Zhaoqing et al. (2013), who was studying "an epidemiological survey of stroke among rural Chinese adults" and stated that the incidence of stroke in rural areas was higher than that found in urban areas and Western countries.

Concerning medical history of the studied patients, the study revealed that two third of the studied patients were having ischemic stroke and this results was supported by Serda et al.,(2015) who stated that $73 \%$ of his sample had ischemic stroke. Also Zaky et al.,(2015) found that $80 \%$ of his study group had ischemic stroke this result also supported by Donnan et al.,(2008) who stated that $85 \%$ of strokes are caused by ischemic while haemorregic strokes represents approximately $15 \%$ of all strokes.

Regarding to previous hospitalization, more than half of the studied patients reported previous hospitalization with stroke. This may be due to non-compliance with stroke preventive measures. This result in the same line with Bedir et al., (2018) who was studying "Effect of nursing educational program on knowledge among patients at risk for stroke "and reported that two third of studied patient had previously hospitalized during last year.

Regarding to risk factors of stroke, it was found that nearly half of patients were having hypertension. This may be due to high blood pressure strains blood vessel walls causing them to thicken and deteriorate and lead to stroke.

This results supported by Khedr et al., (2013) who stated that most of patients had one or more risk factors for stroke, hypertension being the commonest (66\%). Also this results supported by Zaky et al. (2015) who found that 
more than half of study group had hypertension and in the line with Robinson-Smith, Johnston and Allen, (2000) who reported in their study about "Self-care self-efficacy, quality of life, and depression after stroke "that $71 \%$ of their studied sample had hypertension.

In relation to disability from stroke, nearly half of the studied patients were have two or more disabilities like sensory, motor, mental, visual or speech. From the researcher point of view, this may be due to the nature of cerebrovascular accidents which affect many vital centers in brain such as vision, hearing, memory and speaking centers. These results agreed with Abd El-Hay, Abed Allah and Tag El Din (2018), in their study about "Effect of implementing designed educational training program for neurological nurses on clinical outcomes of stroke patients "who found that the majority of patients suffered from; movement disorders, numbness and tingling, symmetrical face, asymmetrical arm weakness, asymmetrical leg weakness, bowel field deficits and speech disorders.

Also these results were in the same line with Ali (2013) who was studying about "Effect of nursing care strategy on the functional and physical abilities of patients following stroke" mentioned that slightly more than one-third of stroke patients having little such as loss in mobility and speech problems which occur following stroke and more than one quarter of stroke patients had visual problems.

In relation to knowledge about definition of stroke, types, risk factors, complication and prevention, the results revealed that low mean score preprogram implementation.The researcher think that the reason for this results may be due to most of patients were illiterate and lived in rural areas where there are lack of awareness about this disease. On the other hand, the mean scores were increased immediate, and on $1^{\text {st }}$ and $3^{\text {rd }}$ monthposteducational program implementation follow up with a high statistical significant difference from pre educational program implementation, although, the mean scores got low among phases of follow up which indicated that the patients with chronic condition need more frequent follow up.

This result agreed with Faiz et al., (2018) who studied "Patient knowledge on stroke risk factors, symptoms and treatment options" and Pandian et al., (2006) who studied "Knowledge of stroke among stroke patients and their relatives in Northwest Indi", found that the level of stroke knowledge in stroke patients seem to be poor. Also this result supported by Wang et al. (2018) who reported in his study about "Acute stroke patients' knowledge of stroke at discharge in China" that the level of knowledge of acute ischemic stroke patients at discharge was not high.

Also this results in the same line with Ibrahim and Soliman (2016) who was studying about "the effect of educational program for high risk people about stroke prevention "stated that there is an improvement with statistically differences in knowledge score pre and post program implementation (Immediate and 2 month post)

This finding is congruent with Feld-Glazman, Bushnik and Van Lew (2012) in their study about "impact of a stroke education program on patient's stroke knowledge and their change of stroke risk behavior" who found that there is a significant improvement in stroke knowledge after completing the education program immediately and at 12 week follow-up.

But this result disagree with Smith, Foster and Young, (2004) who reported in their study about "A randomized trial to evaluate an education program for patients and carers after stroke" that there is no improvement in stroke patients knowledge score post implementing the educational program.

Concerning knowledge related to activity of daily living, the study revealed that there is an increase in the mean score of patients' knowledge immediately post program implementation and during follow up phases $\left(1^{\text {st }}\right.$ and $3^{\text {rd }}$ month) than pre implementation. This result agreed with Hattachot (2017)who was studying "effects of discharge planning program on knowledge and activities of daily living of stroke patients in stroke unit Udonthani Hospital " and reported that an increase in the mean score of knowledge about activity of daily living in study group after implementing discharge planning program than pre implementation.

From the researcher's point of view, this result may be due to health team neglect to provide the patients with health education and information needed to adapt with their disease besides the increased work load and shortage number as well as the lack of time of the health team. Joyce and Jane, (2009) reported that, education is the key to successful treatment of the disease, and the nurse plays a major role as patient educator. Patient and their families need accurate information about the disease and about strategies to minimize its impact. Effective education can alter behavior and empowering patient to make a positive change in their health status.

As regard to activity of daily living for patient with stroke (Barthel scale), the result revealed that approximately two third of patients were severely dependent before implementing discharge planning program but more than half of them were mildly dependent after implementing discharge plan program $\left(3^{\text {rd }}\right.$ month $)$, also the total mean score 
of barthel index categories(BI) were increased and improved post program implementation $\left(1^{\text {st }}\right.$ and $3^{\text {rd }}$ months $)$ $\operatorname{was}(71 \pm 18.05$ and $79.5 \pm 17.21 \mathrm{SD})$ respectively than pre -program implementation $(53.2 \pm 22.71 \mathrm{SD})$ with highly statistically significant differences $(\mathrm{p}<0.001)$. The researcher think this result may be due to patient in the first day of stroke has complete dependence in activity of daily living, but after one month, they return to some extent to their independency with positive impact of a design program implementation.

This result supported by Tveiten et al., (2014), who reported that considerable improvement from acute stroke had taken place during these periods, particularly between the time of discharge and 1 month. This early improvement can be attributed to the spontaneous neurological processes Kong and Lee (2014). This occurs within 3 months after stroke Between 6 months and 2 years post-acute stroke, the Barthel Index score still improves but not considerably. Von Vogelsang, et al., (2015).

This results in the same line with Musa and Keegan (2018), who was studying about "The change of Barthel Index scores from the time of discharge until 3-month post discharge among acute stroke patients in Malaysia" and reported that barthel index score increased from (35.1 \pm 39.4$)$ at time of discharge to $(64.4 \pm 39.5)$ at one month and to $(71.5 \pm 38.9)$ at three month.

This results agreed with Hattachot (2017), who found that an improvement in total mean score of barthel index scale in his experimental group after implementing discharge program $(70.3 \pm 21.3 \mathrm{SD})$ than pre implementation (49.8 \pm 23.5 SD). Also this results supported by Smith, Foster and Young, (2004) and Zaky, et al., (2015) who reported that an increase in score of barthel index after implementing educational program than base line score.

Concerning quality of life for patient, the current study revealed that none of the patients had good QOL at the pre-implementation phase. This is quite expected since these patients were recruited within the first days of the attack, which is a phase of great disablement, full dependence, and lack of adaptation and coping. In congruence with this Langhammer and Stanghelle, (2003) who was studying" Bobath or motor relearning programme? A follow-up one and four years post stroke" reported high levels of physical dependence after stroke.

Also, study revealed that there is an improvement in the total mean score in all domains of quality of life post implementing program on $1^{\text {st }}$ month and $3^{\text {rd }}$ month where the total mean score of SSQoL was $(179.27 \pm 29.66$, $150.44 \pm 39.70)$ respectively than pre implantation $(125.2 \pm 29.88)$ with highly statistically significant differences .These results are consistent with Ali (2013)who reported in his study the implementation of the nursing care strategies proved to be successful in effecting significant and considerable improvements in patients' physical, functional, and total QOL.

Also the result was in the same line with Serda et al., (2015) who demonstrated that all parameters of short form -36 score of studied patient have a significantly improvement than control group and supported by Kamel et al., (2010) who found that there is an improvement in all domains of quality of life after three months.

The results in the same line with Baminidevi, (2019) who was studying about "Comprehensive Stroke Education Program (CSEP) on Knowledge and Quality of Life among Patients with Stroke and Burden among Caregivers" and found that there was an improvement in all 12 domains of SSQoL after one month and three month than preprogram.

In relation to patients' knowledge and level of education the result of the study revealed that the people with university education had high mean score of knowledge pre and post implementing program with highly statistically difference. These results agreed with Wang et al., (2018), Pandian et al (2006) and Yang et al., (2014) who study about "Knowledge of stroke symptoms and treatment among community residents in Western Urban China." reported that mastery of stroke related knowledge was positively associated with educational level.

From the researcher point of view, this result may be due to the patient with high education has awareness about disease from reading posters , article in journal or searching information about diseases in internet.

In relation to patients' knowledge and gender, the result of the study demonstrated that female had high mean score of knowledge than male in pre and post program implementation. The researcher think that the reason for this result may be due to most female was housewife and get there information from watching medical program on television and mass media. These results were in the same line with Wang et al., (2018) who found that the mean score of female knowledge was better than males.

In relation to patients' knowledge and residence, the study revealed that patients who live in urban areas had high mean score of knowledge than who lived in rural areas. These results may be due to lack of medical resources, poor economic condition and relative inaccessibility of medical information in rural areas.

In relation to Sociodemographic and quality of life, the result revealed that the university, female, worked and 
urban patients had high mean score of quality of life with highly statistically differences. These results agreed with Kranciukaite et al., (2007) about "Quality of life in stroke survivors" who demonstrated that HRQOL is correlated with education and employment. These results were disagreed with Gargano and Reeves (2007) who was studying" Sex Differences in Stroke Recovery and Stroke-Specific Quality of Life" found that females had lower SSQOL than males.

In relation to Sociodemographic characteristics and activity of daily living (Barthel index), the result revealed that the university, female, worked and urban patients had high mean score of barthel index with highly statistically differences. This results incongruent with Haghgoo et al., (2013) study about "Depression, activities of daily living and quality of life in patients with stroke", Lai (2005) about" Sex differences in stroke recovery", Gargano and Reeves (2007) and Appelros et al., (2010) study about "A review on sex differences in stroke treatment and outcome" who reported that barthel index score for male better than females.

The present study revealed that there was negative correlation between age and knowledge, quality of life and barthel index which reflect the younger patients have highest knowledge, QOL and barthel index score than older patients. These results supported by, Kamel et al., (2010) and Haacke et al., (2006) who was studying "Long-term outcome after stroke: evaluating health-related quality of life using utility measurements" and Sturm (2004) study about "Quality of life after stroke, the North East Melbourne Stroke Incidence Study" who stated that there is decreasing in HRQOL in older age.

The researcher think that the reason for this result may be due to aging effect on person physically, psychologically, emotionally and socially that in turn leads to decrease QOL. It was likely that with the increasing age, the body becomes weaker, making recovery slower, brain tissue becomes damaged, and the protective effects of the endothelium and astrocytes in the brain are dysregulated with a consequent negative effect on the sensory-motor recovery (Sohrabji, Bake and Lewis,2013).

In contrast Carod-Artal, (2000) studied about "Quality of life among stroke survivors evaluated 1 year after stroke, experience of a stroke unit "found that older age wasn't correlated with low QOL, also Patel et al., (2007) in his study about "clinical determinants of long -term quality of life after stroke" showed that younger subjects reported worse HRQOL .

Also the results showed that there was a positive correlation between knowledge, barthel index and quality of life as well as between QOL and barthel index along pre and post program implementation with high statistical significant differences. These results supported by Zaky et al., (2015) who showed that there was a positive statistical significant correlation between barthel index and knowledge (high knowledge high Barthel). Also these results agreed with Haghgoo et al., (2013) who reported better performance in activity of daily living was correlated with higher score in QOL.

\section{Conclusion}

Based on the findings of the current study, it can be concluded that,

The post mean knowledge, quality of life and activity of daily living scores of patients with stroke who were exposed to a design discharge planning program were higher than their pretest mean scores. There were positive relations between patient's knowledge, quality of life, activity of daily living and with their socio demographic characteristics.

\section{Recommendations}

Based on results of the present study the following can be recommended:

- There is a need for continuous monitoring and evaluating knowledge, quality of life, activity of daily living of stroke patients for early detecting and solving any problems.

- Implementing required policies and programs are recommended regarding the establishment of discharge planning program for stroke patients.

- Apply study with large exploration to evaluate and monitoring physical problems affecting on the quality of life and activity of daily living for patients with stroke.

- Reapply this research on large probability sample from different geographical areas in Egypt for generalization. 


\section{Acknowledgement}

The researchers would like to extend their great appreciation to all health personnel in the stroke department and stroke outpatient clinics of Benha University Hospital as well as the patients involved in the study for their cooperation in the fulfillment of this study.

\section{References}

Abd El-Hay, S., Abed Allah, A., \& Tag El Din, E. (2018). Effect of implementing designed educational training program for neurological nurses on clinical outcomes of stroke patients. Clinical Nursing Studies, 6(4), 121-137.

Ali, Z. H. (2013). Effect of Nursing Care Strategy on the Functional and Physical Abilities of Patients Following Stroke. Journal Neuro l Neurophysiology, 8, 6 .

Appelros, P., Stegmayr, B., \& Terent, A. (2010). A review on sex differences in stroke treatment and outcome. Acta Neurol Scand, 121(6), 359-69.

Baminidevi, N. (2019). Comprehensive Stroke Education Program (CSEP) on knowledge and quality of life among patients with stroke and burden among caregivers. Nursing and Health Care, 4, 71-80.

Bedir, N., Sheir, M., Shahin, E., \& Abd El-Reheem, H. (2018). Effect of nursing educational program on knowledge among patients at risk for stroke. Port Said Scientific Journal of Nursing, 5(1), 188-211.

Boosman, H., Passier, P., Visser-Meily, J., Rinkel, G., \& Post, M. (2010). Validation of the Stroke Specific Quality of Life scale in patients with aneurysmal subarachnoid haemorrhage. Journal Neurol Neurosurg Psychiatry, 81(5), 485-9.

Capistrant, B., Wang, Q., Liu, S., \& Glymour, M. (2013). Stroke-associated differences in rates of activity of daily living loss emerge years before stroke onset. Journal of the American Geriatrics Society, 61(6), 931-938.

Carod-Artal, F., \& Egido, J. (2009). Quality of life after stroke: The importance of a good recovery. Cerebrovascular Diseases, 27(Suppl. 1), 204-214.

Carod-Artal, J., Egido, J., Gonzalez, J., \& Seijas, V. (2000). Quality of life among stroke survivors evaluated 1year after stroke, experience of a stroke unit. Stroke, 31, 2995-3000.

Donnan, G., Fisher, M., Macleod, M., \& Davis, S. (2008). Stroke. Neurology Journal, 12, 161-22.

Eftekhar, P., Cameron, D., Yoshida, K., Landry, M., \& Brooks, D. (2010). Clients' and Health Care Professionals' Perspectives on Post Stroke Rehabilitation Services in Tehran, Iran. Middle East Journal in Age and Ageing, $7(1), 33-40$.

Egyptian ministry of health. (2017). 5th MENA Conference in Cairo. Retrieved from https://eso-stroke.org/strokeresearch/5th-mena-conference-cairo-18-20-october-2017/

Faiz, K., Sundseth, A., Thommessen, B., \& Ronning, O. (2018). Patient knowledge on stroke risk factors, symptoms and treatment options. Vasc Health Risk Manag, 14, 37-40.

Feigin, V. L. (2013). Global and regional burden of stroke findings from the global burden of disease study. Lancet, $383,245-255$.

Feld-Glazman, R., Bushnik, T., \& Van Lew, S. (2012). The Impact of a Stroke Education Program on Patient's Stroke Knowledge and Their Change of Stroke Risk Behavior. Physical medicine and rehabilitation, 93(10), 39.

Gargano, J., \& Reeves, M. (2007). Sex Differences in Stroke Recovery and Stroke-Specific Quality of Life. Stroke, 38, 2541-2548. Retrieved June 23, 2019, from http://ahajournals.org

Haacke, C., Althaus, A., Spottke, A., Siebert, U., Back, T., \& Dodel, R. (2006). Long-term outcome after stroke: evaluating health-related quality of life using utility measurements. Stroke, 37, 193-8.

Haghgoo, H., Pazuki, E., Hosseini, A., \& Rassafiani, M. (2013). Depression, activities of daily living and quality of life in patients with stroke. Journal of the Neurological Sciences, 328, 87-91.

Hattachot, Y. (2017). Effects of Discharge Planning program on Knowledge and Activities of Daily Living of Stroke Patients in Stroke Unit Udonthani Hospital. Udonthani Hospital Medical Journal, 25(2), 136-142.

Ibrahim, R., \& Soliman, F. (2016). The Effect of Educational Program for High Risk People about Stroke Prevention. Journal of Nursing and Health Science, 5(4), 83-89.

Jauch, E., Saver, J., Adams, H., Bruno, A., Connors, J., Demaerschalk, B., \& Yonas, H. (2013). Guidelines for the 
early management of patients with acute ischemic stroke: A guideline for healthcare professionals from the American Heart Association/American Stroke Association. Stroke, 44(3), 870-947.

Joyce, B., \& Jane, H. (2009). Medical Surgical Nursing. Congress (8th ed.).

Kamel, A., Abdel Ghani, A., Zaiton, M., El-Motayam, A., \& El Fattah, D. (2010). Health Related Quality of Life in Stroke Survivors Measured by the Stroke Impact Scale. Egypt Journal Neurol Psychiat Neurosurg, 47(2), 267-274.

Khedr, E., Elfetoh, N., Al Attar, G., Ahmed, M., Ali, A., Hamdy, A., Kandil, M., \& Farweez, H. (2013). Epidemiological Study and Risk Factors of Stroke in Assiut Governorate, Egypt: Community-Based Study. Neuroepidemiology, 40, 288-294.

Koenig-Bruhin, M., Kolonko, B., At, A., Annoni, J. M., \& Hunziker, E. (2013). Aphasia following a stroke: Recovery and recommendations for rehabilitation. Swiss Archives of Neurology and Psychiatry, 164(8), 292-298.

Kong, K., \& Lee, J. (2014). Temporal recovery of activities of daily living in the first year after ischemic stroke: a prospective study of patients admitted to a rehabilitation unit. Neuro Rehabilitation, 35(2), 221-6.

Kothari, R., Sauerbeck, L., Jauch, E., Broderick, J., Brott, T., \& Khoury, J. (1997). Patient's awareness of stroke signs, symptoms and risk factors. Stroke, 28, 1871-5.

Kranciukaite, D., Rastenyte, D., Jureniene, K., \& Sopagiene, D. (2007). Quality of life in stroke survivors. Medicina (Kaunas), 43, 736-45.

Lai, S., Duncan, P., Dew, P., \& Keighley, J. (2005). Sex differences in stroke recovery. Prev Chronic Dis, 2(3), A13.

Langhammer, B., \& Stanghelle, J. (2003). Bobath or motor relearning programme? A follow-up one and four years post stroke. Clin Rehabil, 17, 731-734.

Maslakpak, M., \& Shams, S. (2015). A comparison of face to face and video-based self-care education on quality of life of hemodialysis patients. International Journal of Community Based Nursing and Midwifery, 3(3), $234-243$.

Musa, K., \& Keegan, T. (2018). The change of Barthel Index scores from the time of discharge until 3-month post discharge among acute stroke patients in Malaysia: A random intercept model. Plos One, 13(12), e0208594.

Muus, I., Williams, L., \& Ringsberg, K. (2007). Validation of the Stroke Specific Quality of Life Scale (SS-QOL): test of reliability and validity of the Danish version (SS-QOL-DK). ClinRehabil, 21(7), 620-7.

National Stroke Association - NSA. (2016). U.S. Department of Health and Human Services.

National Stroke Foundation. (2011). Clinical guidelines for stroke management. Retrieved from https://strokefoundation.com.au/ /media/strokewebsite/resources/treatment/

Ostwald, S. K. (2015). Predictors of life satisfaction among stroke survivors and spousal caregivers: a narrative review. Journal of Aging Health, 4(3), 241-252.

Pancioli, A., Broderick, J., Kothari, R., Brott, J., Tuchfarber, A., \& Miller, R. (1998). Public perception of stroke warning signs and knowledge of potential risk factors. JAMA, 279, 1288-92.

Pandian, J., Kalra, C., Jaison, A., Deepak, S., Shamsher, S., Singh, S., \& Abraham, G. (2006). Knowledge of stroke among stroke patients and their relatives in Northwest Indi. Neurology India, 54(2), 152-156.

Patel, M., Mckevitt, C., Lawrence, E., Rudd, C., \& Wolfe, A. (2007). Clinical determinants of long -term quality of life after stroke. Age and Ageing, 36(3), 316-22.

Peoples, H., Satink, T., \& Steultjens, E. (2011). Stroke survivors' experiences of rehabilitation: A systematic review of qualitative studies. Scandinavian Journal of Occupational Therapy, 18(3), 163-171.

Robinson-Smith, G., Johnston, M., \& Allen, J. (2000). Self-care self-efficacy, quality of life, and depression after stroke. Arch Phys Med Rehabil, 81, 460-4.

Sacco, R., Kasner, S., Broderick, J., Caplan, L., Connors, J., Culebras, A., \& Vinters, H. (2013). An updated definition of stroke for the 21st century: A statement for healthcare professionals. Stroke, 44(7), 2064-2089.

Seana, L., Pham, L., Kara, M., Leigh, B., \& Velandai, S. (2012). Sex Differences in Long-Term Outcomes After Stroke Functional Outcomes, Handicap, and Quality of Life. American Heart Stroke Association Journal, 8, 1982-19873 
Serda, M., Bozkurt, M., Karakoç, M., Çağlayan, M., Akdeniz, D., Oktayoğlu, P., Varol, S., \& Nas, K. (2015). Determining of Life Quality and Associated Factors in Stroke Patients. Turk Journal Phys Med Rehab, 61, 148-54.

Smith, J., Forster, A., \& Young, J. (2004). A randomized trial to evaluate an education program for patients and carers after stroke. ClinicalRehabilitation, 18, 726-736.

Sohrabji, F., Bake, S., \& Lewis, D. (2013). Age-related changes in brain support cells: Implications for stroke severity. Neurochemistry international, 63(4), 291-301.

Sturm, J., Donnan, G., Dewey, M., Macdonell, R., Gilligan, A., \& Srikanth, V. (2004). Quality of life after stroke, the North East Melbourne Stroke Incidence Study (NEMESIS). Stroke, 35(10), 2340-5.

Sullivan, O., Susan, B. S., \& Thomas, J. (2007). Physical Rehabilitation (5th ed.). Philadelphia, PA: F.A. Davis Company.

Tveiten, A., Ljostad, U., Mygland, A., \& Naess, H. (2014). Functioning of long-term survivors of first-ever intracerebral hemorrhage. Acta neurologica Scandinavica, 129(4), 269-75.

Von Vogelsang, A., Forsberg, C., Svensson, M., \& Wengstrom, Y. (2015). Patients experience high levels of anxiety 2 years following aneurysmal subarachnoid hemorrhage. World neurosurgery, 83(6), 1090-7.

Wang, M., Mao, L., Xia, Y., He, Q., Lu, Z., Yin, X., \& Bo, H. (2018). Acute stroke patients' knowledge of stroke at discharge in China: a cross-sectional study. Tropical Medicine and International Health, 23(11), 1200-1206.

White, C. L., Barrientos, R., \& Dunn, K. (2014). Dimensions of uncertainty after stroke: Perspectives of the stroke survivor and family caregiver. The Journal of Neuroscience Nursing, 46(4), 233-240.

White, J. H., Magin, P., Attia, J., Sturm, J., Carter, G., \& Pollack, M. (2012). Trajectories of psychological distress after stroke. Annals of Family Medicine, 10(5), 435-442.

Williams, L., Weinberger, M., Harris, L., Clark, D., \& Biller, J. (1999). Development of a stroke-septic quality of life scale. Stroke, 30(7), 1362-9.

Woodman, P., Riazi, A., Pereira, C., \& Jones, F. (2014). Social participation post stroke: A meta-ethnographic review of the experiences and views of community dwelling stroke survivors. Disability and Rehabilitation, 36(24), 2031-2043.

World Health Organization. (2016a). The 10 leading causes of death by country income group (2012). Retrieved from http://www.who.int/mediacentre/factsheets/fs310/en/index1.html

World Health Organization. (2016b). WHO Steps stroke manual. Retrieved from http://www.who.int/chp/steps/Manual.pdf

Yang, J., Zheng, M., \& Cheng, S. (2014). Knowledge of stroke symptoms and treatment among community residents in Western Urban China. Journal Stroke Cerebrovasc Disvo, l23, 1216-1224.

Zaky, H., Mohammad, Z., EL-Labban, A., \& Ahmed, G. (2015). Strategies of Daily Living Rehabilitative Activities for Post Stroke Patients at Minia University Hospital. Journal of Education and Practice, 6(5), 61-72.

Zhaoqing, S., Liqiang, Z., Robert, D., Xingang, Z., Jue, L., Dayi, H., \& Yingxian, S. (2013). An Epidemiological Survey of Stroke among Rural Chinese Adults Results from the Liaoning Province. International Journal of Stroke, 44, 1500-1504.

Ziki, C., DivjakI, J., \& Cevi, C. (2017). The effect of outcome and quality of life. Acta Clin Croat, 53, $294-301$.

\section{Copyrights}

Copyright for this article is retained by the author(s), with first publication rights granted to the journal.

This is an open-access article distributed under the terms and conditions of the Creative Commons Attribution license (http://creativecommons.org/licenses/by/4.0/). 